\title{
Bioethica
}

Vol 5, No 1 (2019)

Bioethica

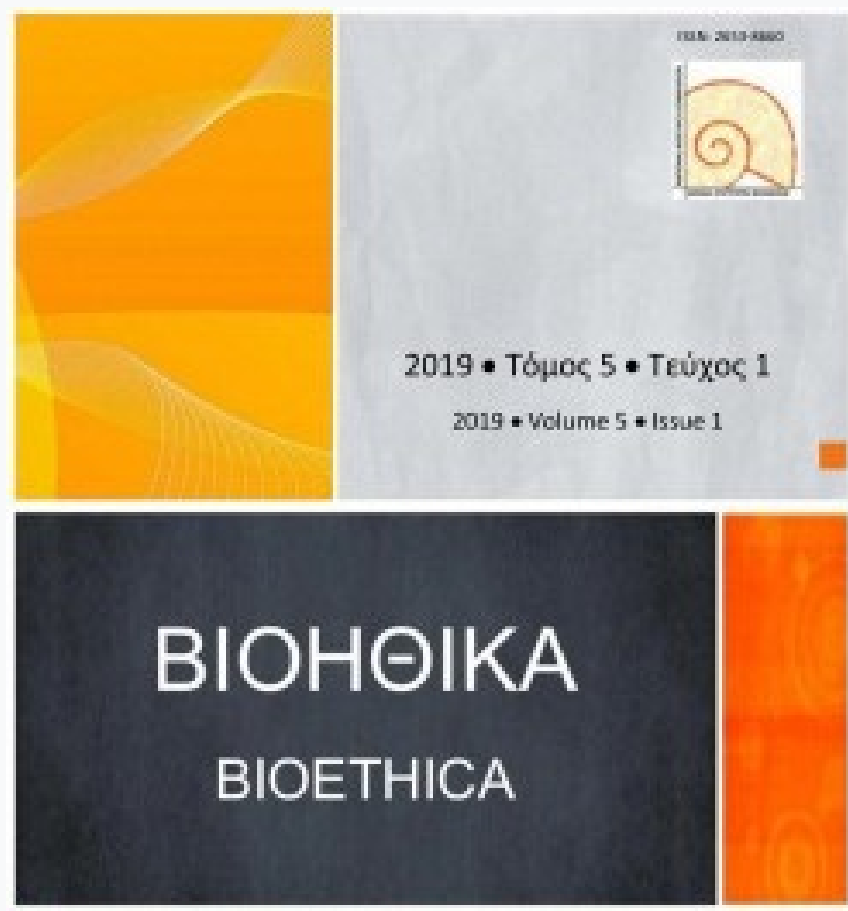

\section{Bioethics in Education}

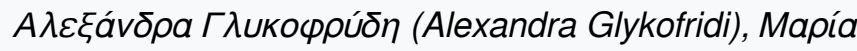
Zanouvíסou (Maria Zapounidou)

doi: $10.12681 /$ bioeth.20833

\section{Copyright ( ) 2019, Alexandra Glykofridi, Maria Zapounidou}

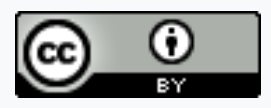

This work is licensed under a Creative Commons Attribution 4.0.

\section{To cite this article:}

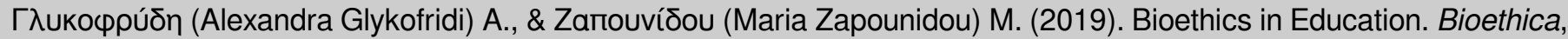
5(1), 13-29. https://doi.org/10.12681/bioeth.20833 


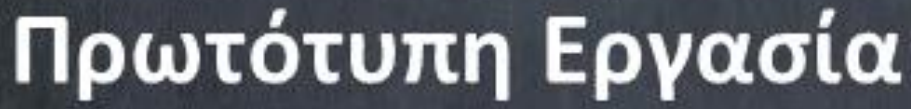

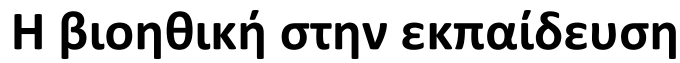

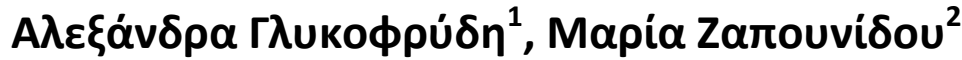

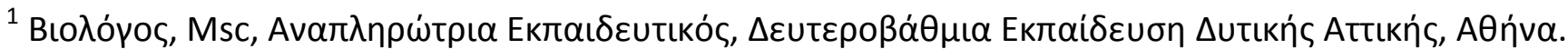

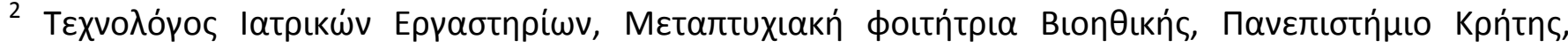

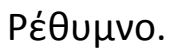

mariazapounidou4@gmail.com

\section{Пєрí $\eta \psi \eta$}

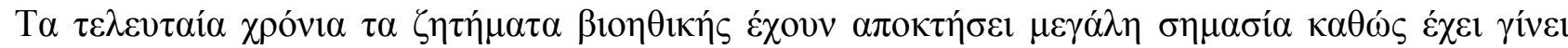

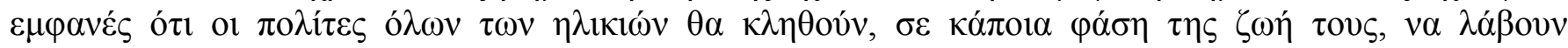

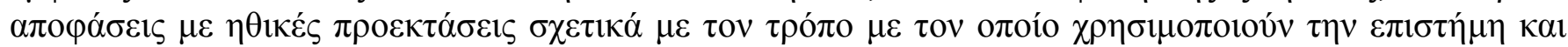

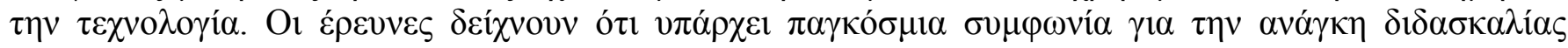

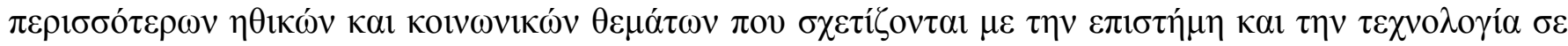
ó $\lambda \varepsilon \varsigma \tau \imath \varsigma \beta \alpha \theta \mu i ́ \delta \varepsilon \varsigma \varepsilon \kappa \pi \alpha i ́ \delta \varepsilon v \sigma \eta \varsigma$.

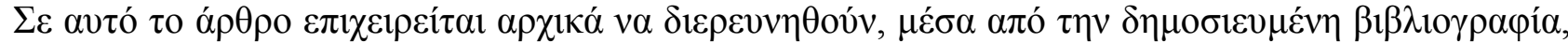

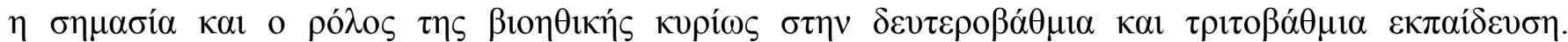

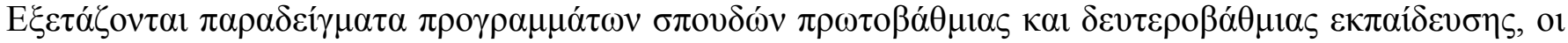

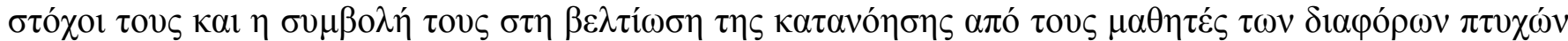

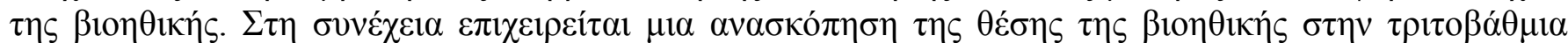

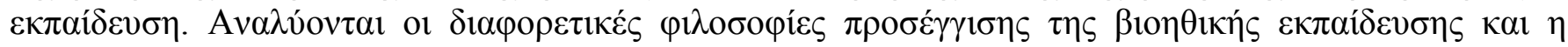

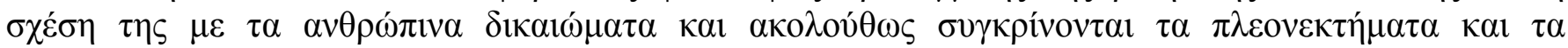

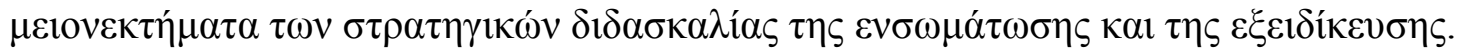

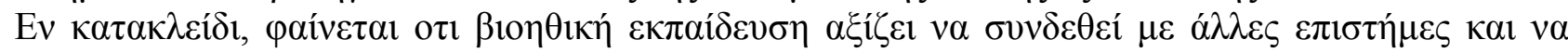

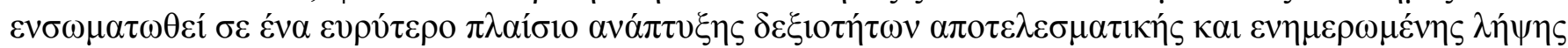

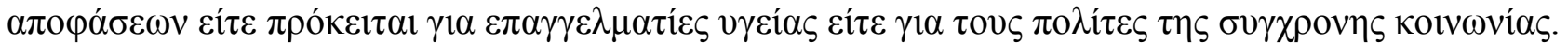

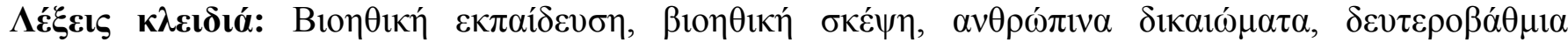
$\varepsilon \kappa \pi \alpha i ́ \delta \varepsilon v \sigma \eta, \pi \alpha v \varepsilon \pi 1 \sigma \tau \eta \mu 1 \alpha \kappa \eta ́ ~ \varepsilon \kappa \pi \alpha i ́ \delta \varepsilon v \sigma \eta, \varepsilon v \sigma \omega \mu \alpha ́ \tau \omega \sigma \eta, \varepsilon \xi \varepsilon 1 \delta i ́ \kappa \varepsilon v \sigma \eta$. 


\title{
Bioethics in Education
}

\author{
Alexandra Glykofridi, ${ }^{1}$ Maria Zapounidou ${ }^{2}$ \\ ${ }^{1}$ Biologist Msc, Supply teacher in Secondary Education in Western Attica, Athens. \\ ${ }^{2}$ Biomedical scientist, Postgraduate student of Bioethics, University of Crete.
}

\begin{abstract}
During recent years, bioethics have become increasingly important as it has become clear that citizens of all ages will be called upon to take ethical decisions about the use of science and technology at some stage of their lives. Research shows that there is a global agreement on the need to teach more of these ethical and social issues related to science and technology at all levels of education.

This article attempts to investigate the importance and role of bioethics in secondary and undergraduate university education. Examples of primary and secondary education curricula, their goals and their contribution to improving students' understanding of the different aspects of bioethics are presented. Then, it is attempted to review the situation of bioethics in universities curricula. The article analyzes the different philosophies of approaching bioethical education, its relation with human rights and compares the advantages and disadvantages of the teaching strategies of integration and specialization.

In conclusion, it seems that bioethics is worthwhile being joined together with other disciplines and integrated into a wider framework of effective and informed decision-making skills, whether the person is a health professional or a modern citizen.
\end{abstract}

Keywords: Bioethics education, bioethical thinking, human rights, secondary education, higher education, integration, specialization. 


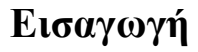

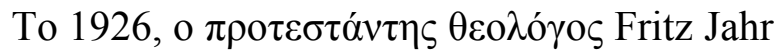

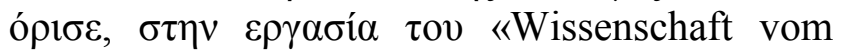

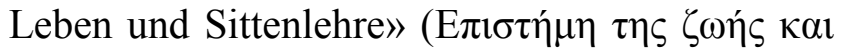

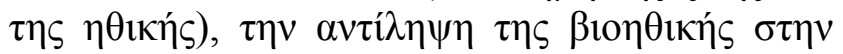

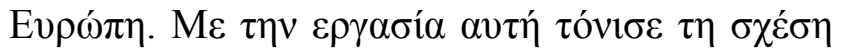

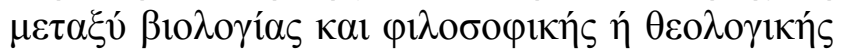

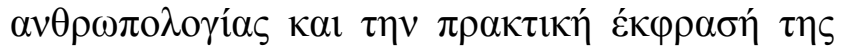

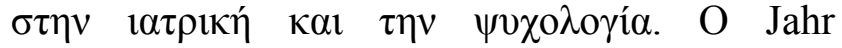

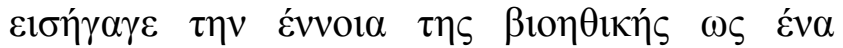

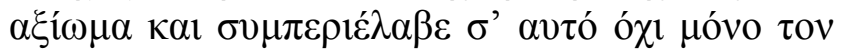

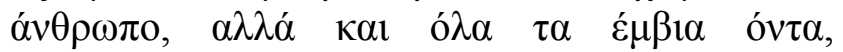

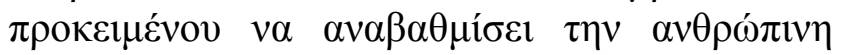

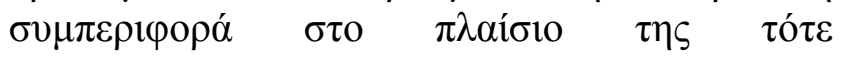

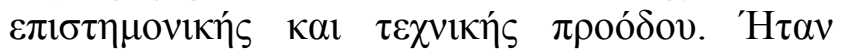

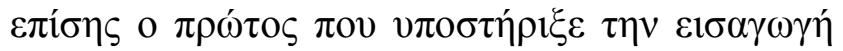
$\tau \omega v \quad \eta \theta \imath \kappa \omega ́ v ~ \alpha \xi ı \omega ́ v ~ \sigma \tau \eta v ~ \delta i \delta \alpha \sigma \kappa \alpha \lambda i ́ \alpha \quad \tau \omega v$

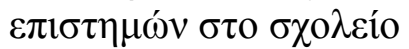

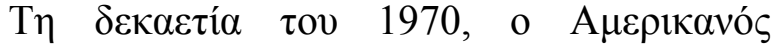

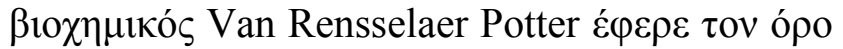

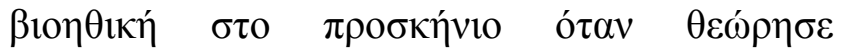

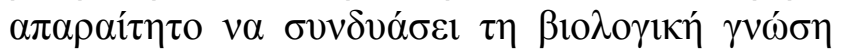

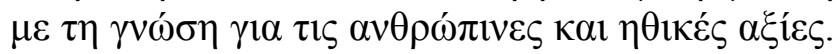

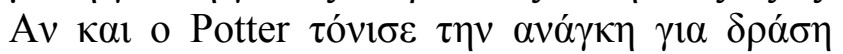

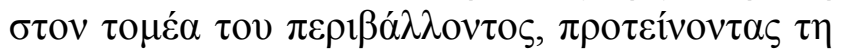

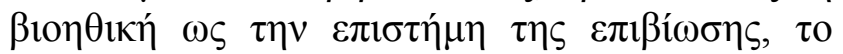

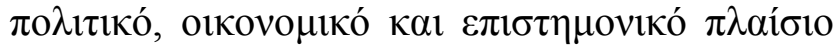

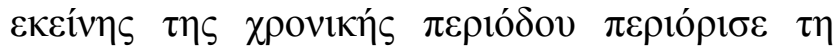

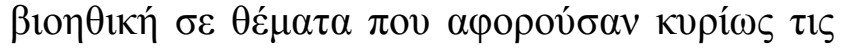

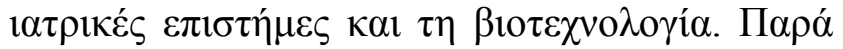

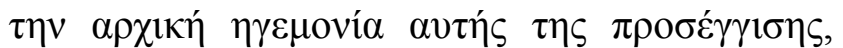

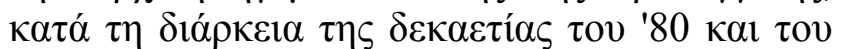

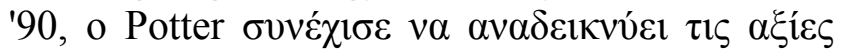

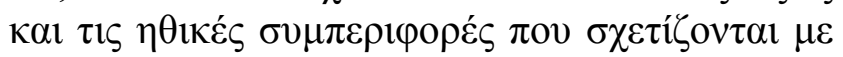

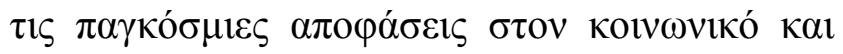

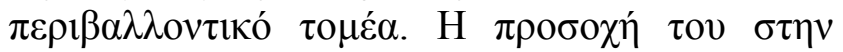

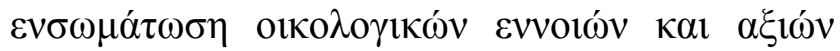

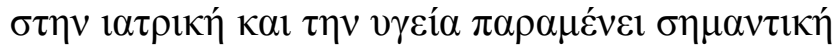

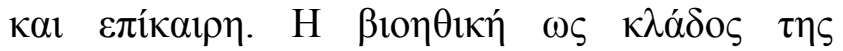

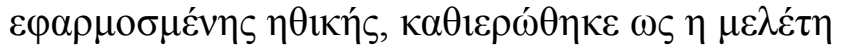

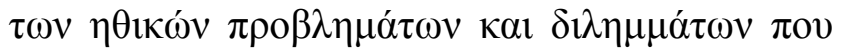

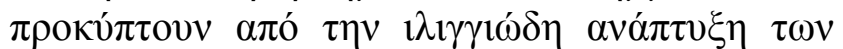

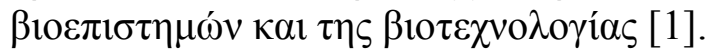

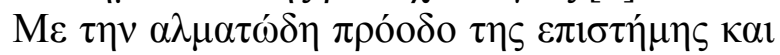

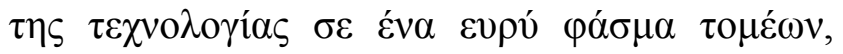
$\sigma v \mu \pi \varepsilon \rho 1 \lambda \alpha \mu \beta \alpha v o \mu \varepsilon ́ v \omega v \quad \gamma 1 \alpha \quad \pi \alpha \rho \alpha ́ \delta \varepsilon \imath \gamma \mu \alpha, \tau \eta \varsigma$

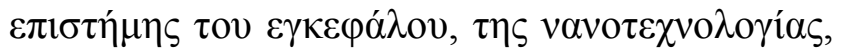

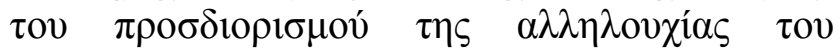

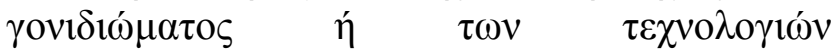

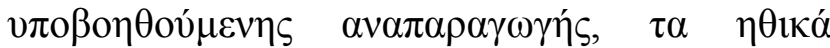

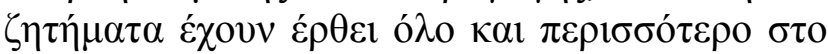

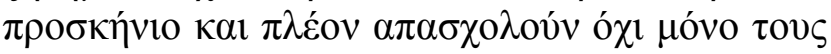

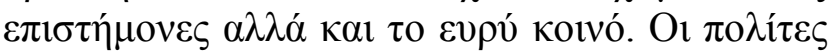

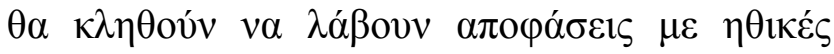

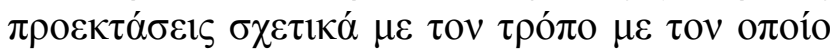

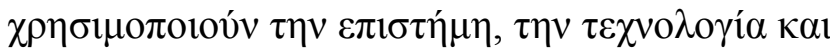

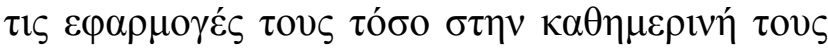

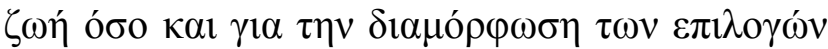

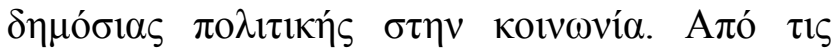

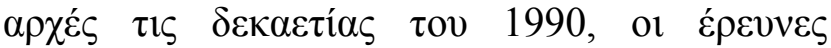

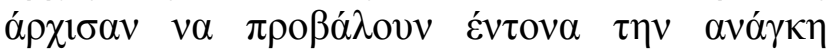
$\delta 1 \delta \alpha \sigma \kappa \alpha \lambda i ́ \alpha \varsigma \quad \pi \varepsilon \rho 1 \sigma \sigma o ́ \tau \varepsilon \rho \omega \nu \quad \eta \theta 1 \kappa \omega ́ v \quad \kappa \alpha 1$

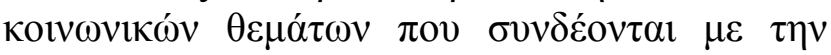

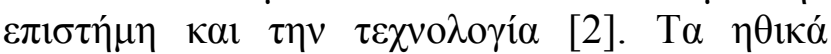
$\zeta \eta \tau \eta ́ \mu \alpha \tau \alpha \tau \omega v \beta 10 \varepsilon \pi ı \tau \tau \eta \omega \omega v ~ \alpha \pi \varepsilon ́ \kappa \tau \eta \sigma \alpha v ~ o \lambda o \varepsilon ́ v \alpha$

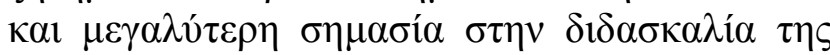

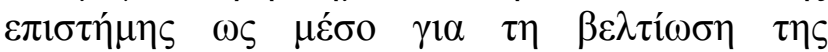

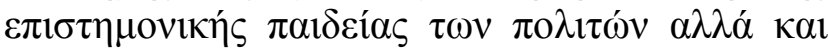

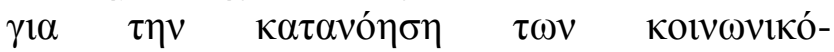

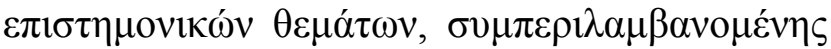

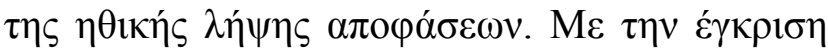

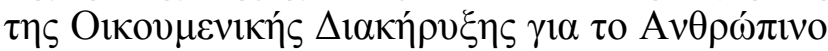

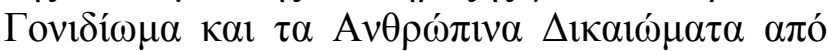

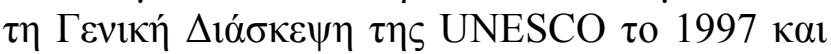

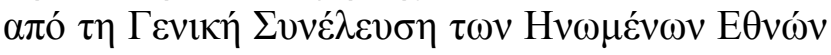

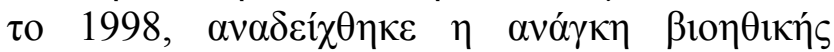

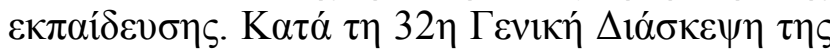

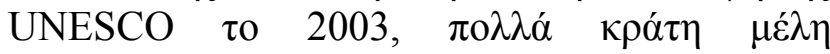
$\varepsilon \xi \dot{\varepsilon} \varphi \rho \alpha \sigma \alpha \nu \quad \tau \eta v \quad \alpha v \alpha ́ \gamma \kappa \eta \quad \delta i \delta \alpha \sigma \kappa \alpha \lambda i ́ \alpha$

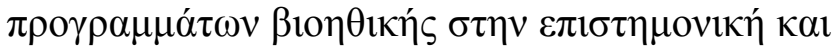

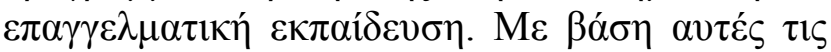

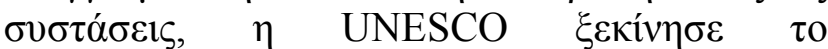

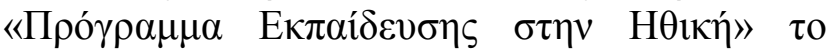

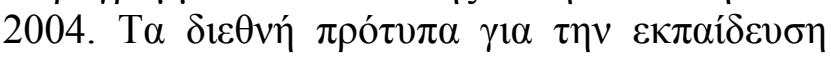

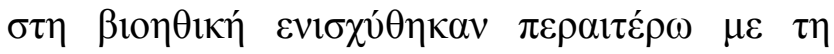

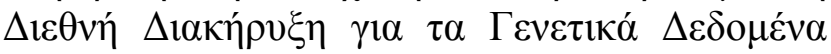

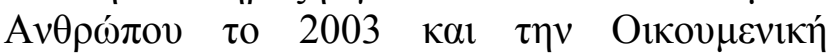

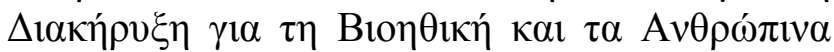

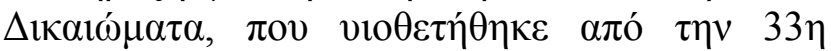

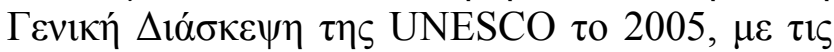

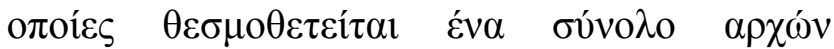

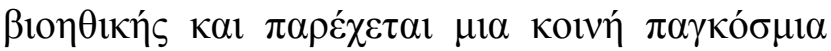

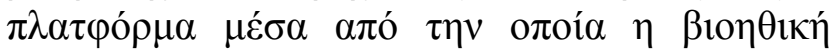

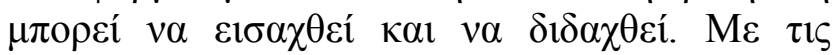

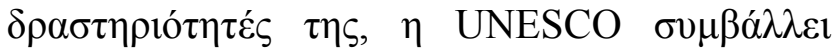

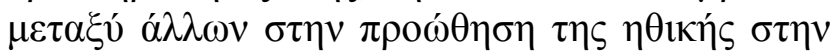

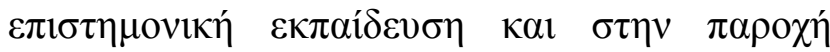

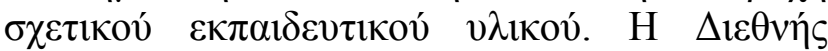

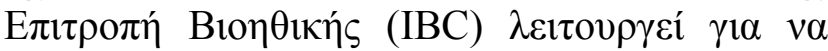




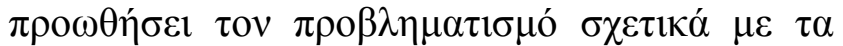

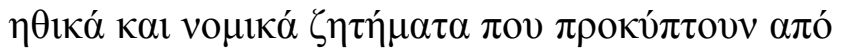

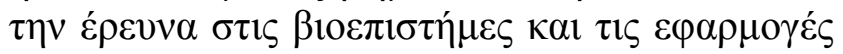

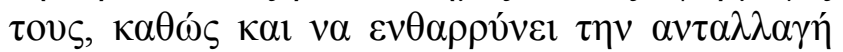

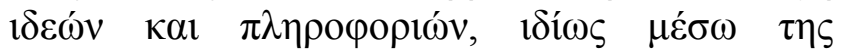

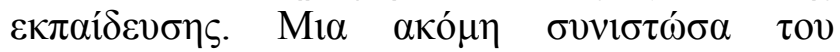

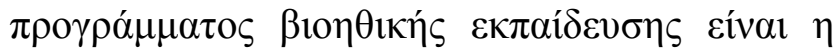

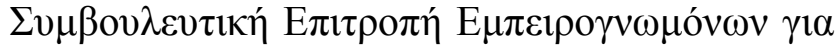

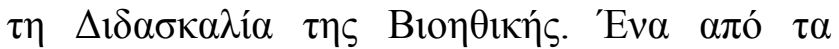

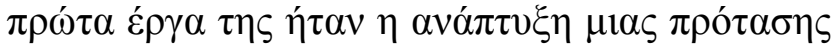

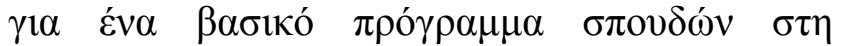

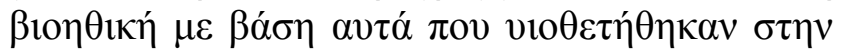

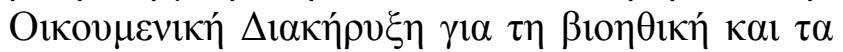

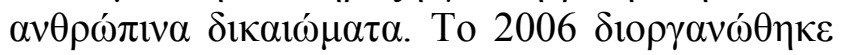

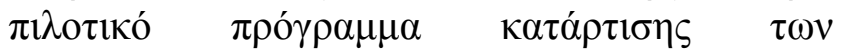

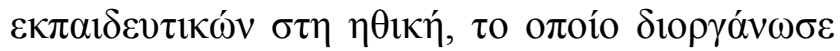
$\eta \varepsilon \dot{\varepsilon} \delta \rho \alpha \tau \varsigma \beta 10 \eta \theta 1 \kappa \eta ́ \varsigma \tau \eta \varsigma$ UNESCO $\sigma \tau \eta$ Haifa

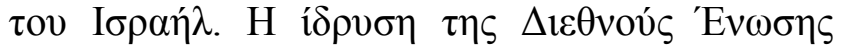

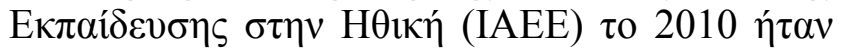

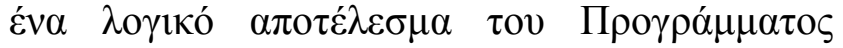

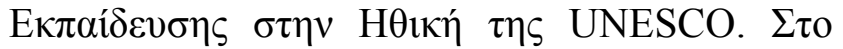

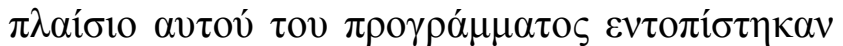

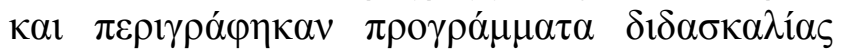

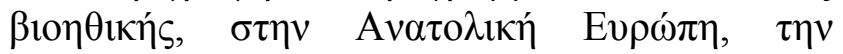

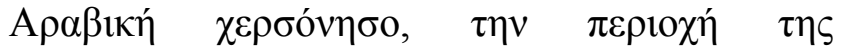

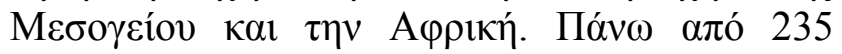

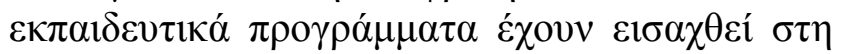

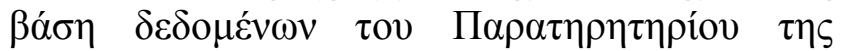

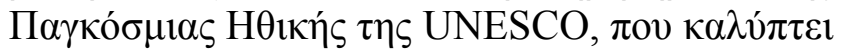

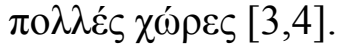

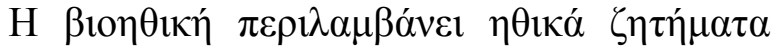

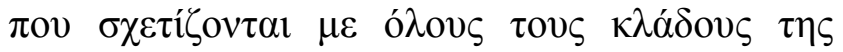

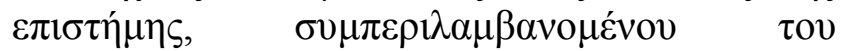

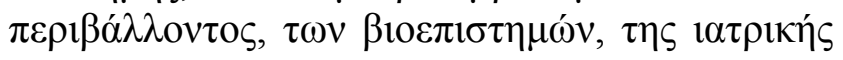

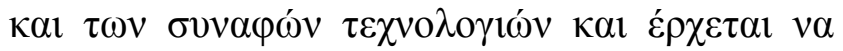

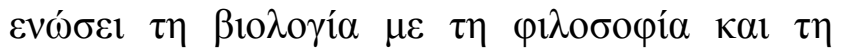

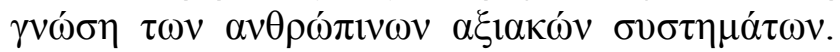

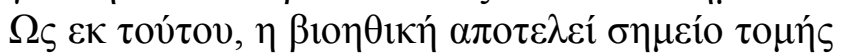

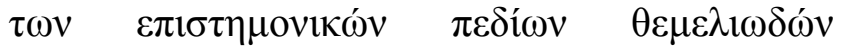

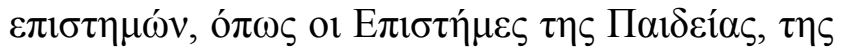

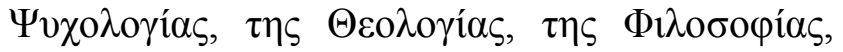

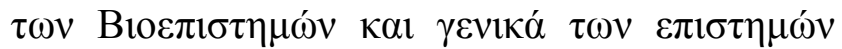

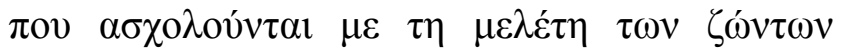

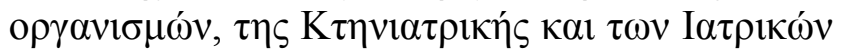

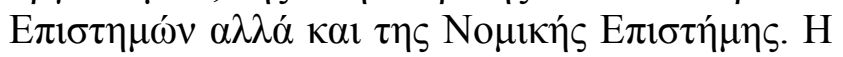

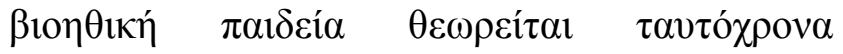

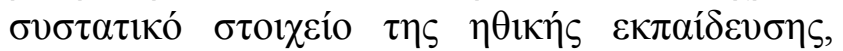

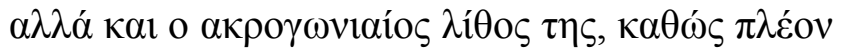

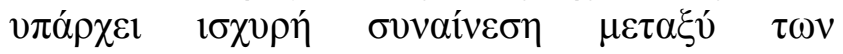

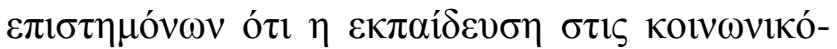

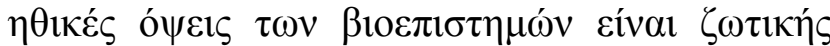

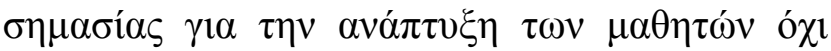

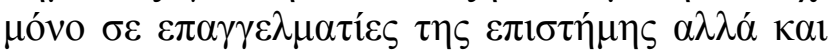

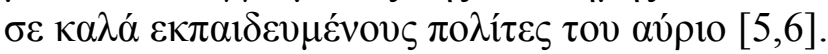

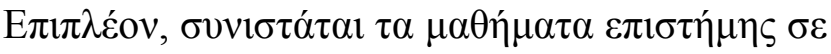
ó $\lambda \alpha \tau \alpha \varepsilon \pi i \pi \varepsilon \delta \alpha \varepsilon \kappa \pi \alpha i ́ \delta \varepsilon v \sigma \eta \varsigma$ v $\alpha \varepsilon v \sigma \omega \mu \alpha \tau \omega ́ v o v v$

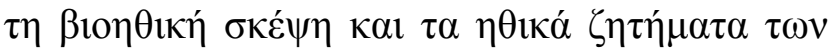

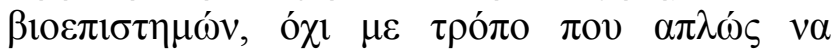
$\varepsilon \pi 1 \sigma \eta \mu \alpha i ́ v o v v \quad \tau \alpha \quad \pi \rho \circ \beta \lambda \eta ́ \mu \alpha \tau \alpha \quad \alpha \lambda \lambda \alpha \quad v \alpha$

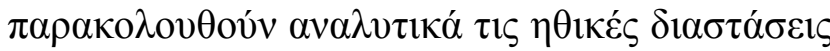

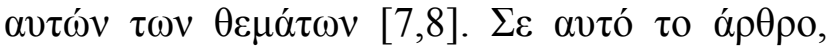

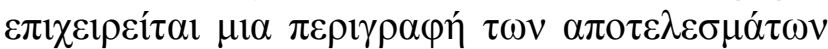

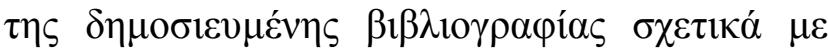

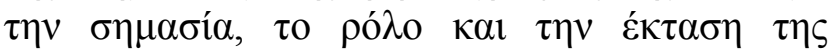

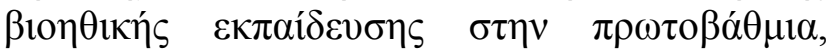

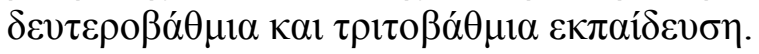

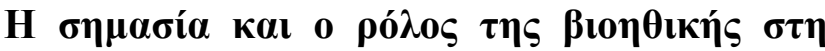

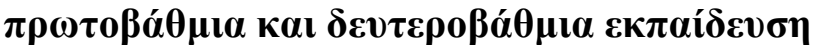

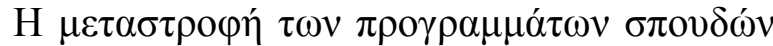

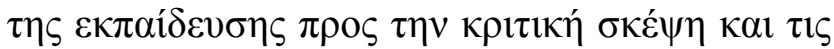

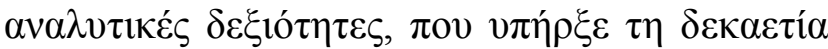

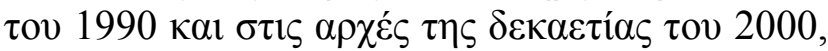

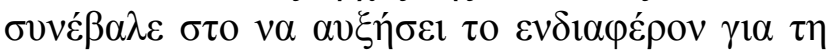

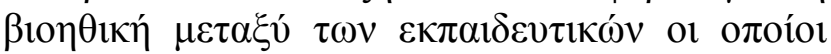

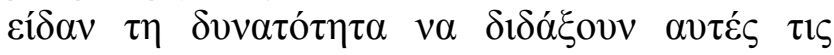

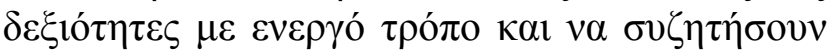

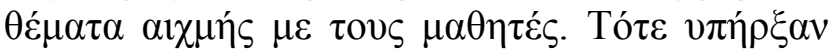

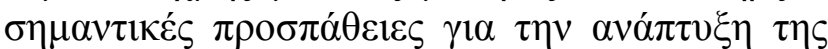

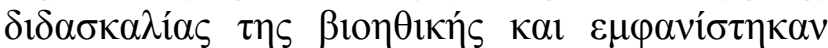

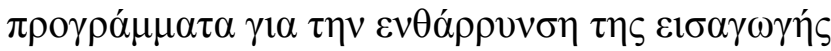

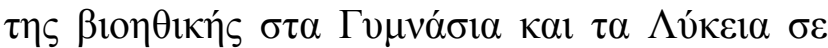

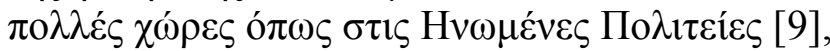

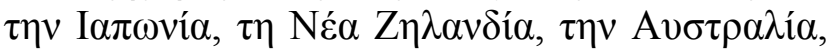

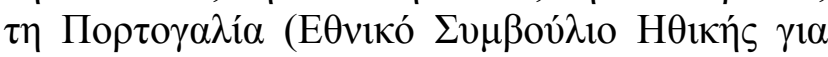

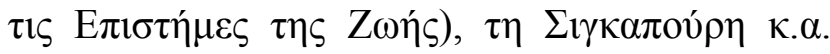
[10].

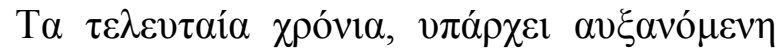

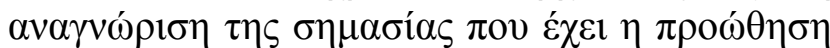

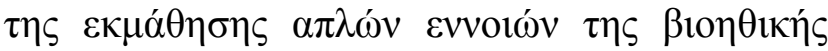

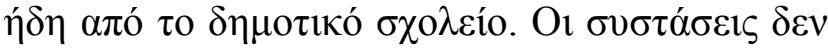

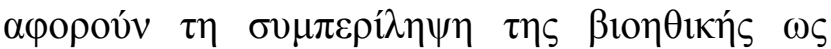

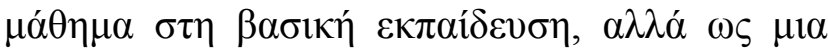

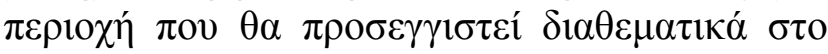

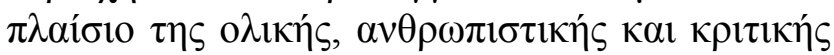

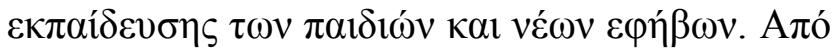

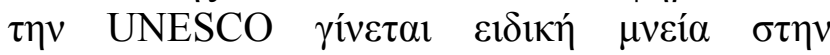

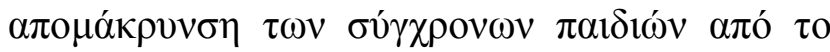

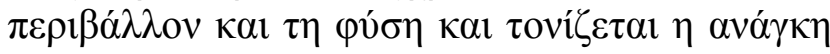

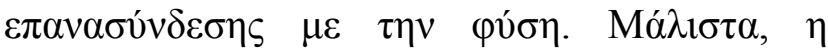




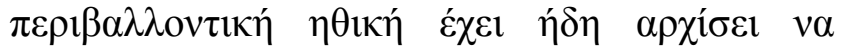

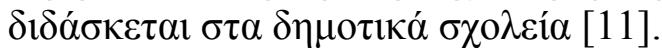

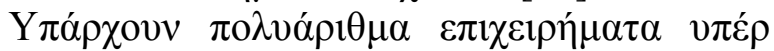

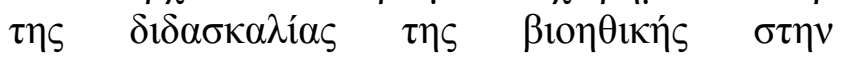

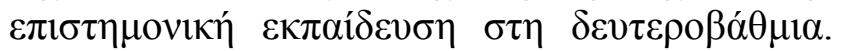

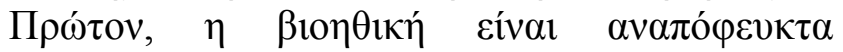

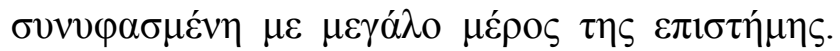

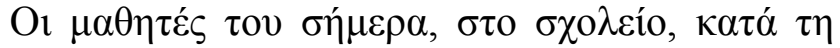

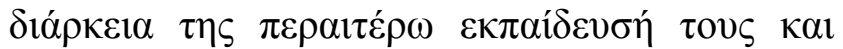

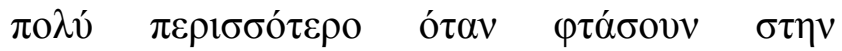

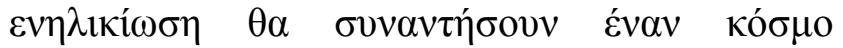

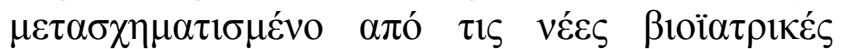

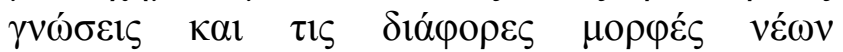

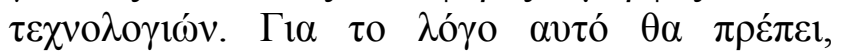

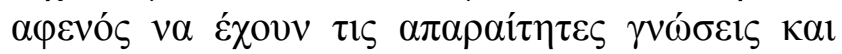

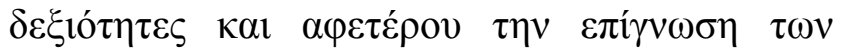

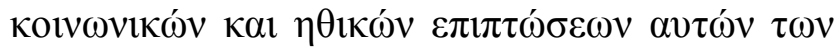

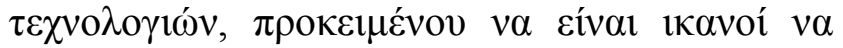

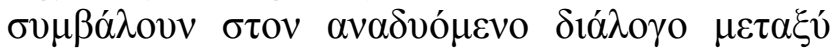

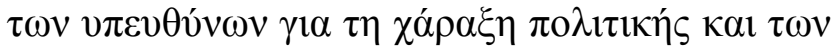

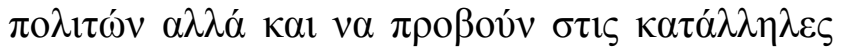

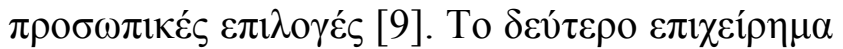

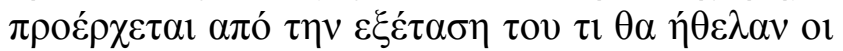
$\mu \alpha \theta \eta \tau \dot{\varepsilon} \zeta \quad v \alpha \quad \delta 1 \delta \alpha ́ \sigma \kappa o v \tau \alpha \imath \quad \sigma \tau \alpha \quad \mu \alpha \theta \eta \dot{\eta} \mu \alpha \alpha \quad \tau \eta \varsigma$

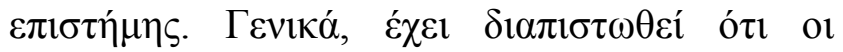

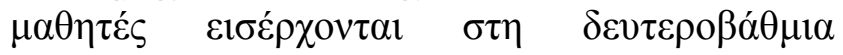

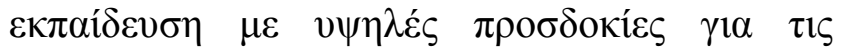

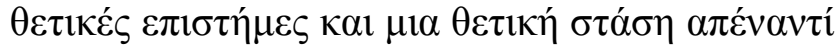

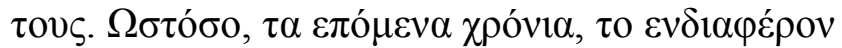

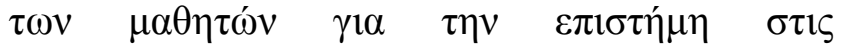

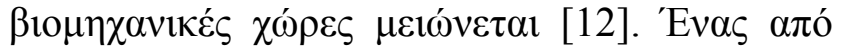

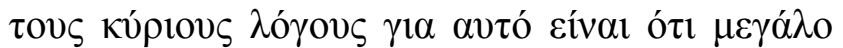

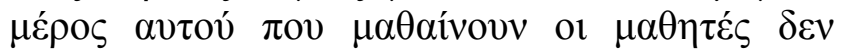

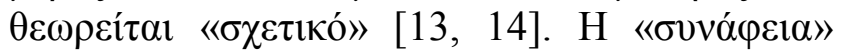

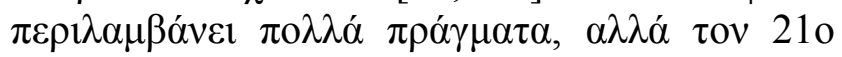

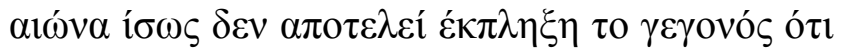

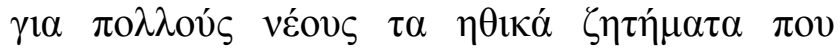

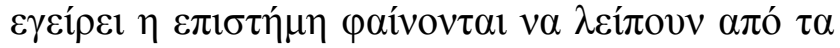

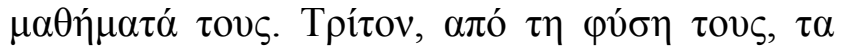

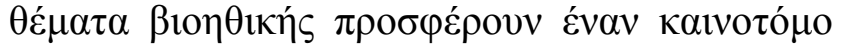

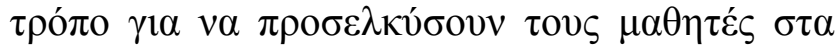

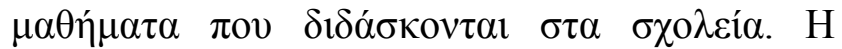

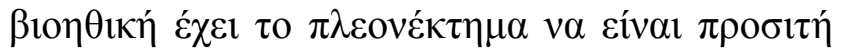

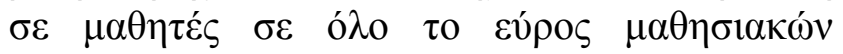

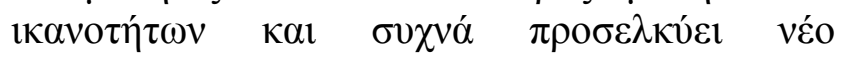

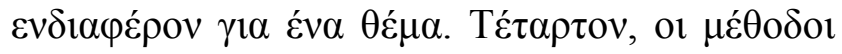

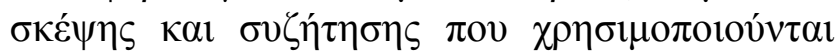

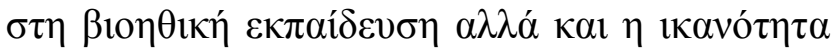

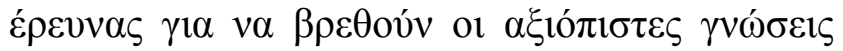

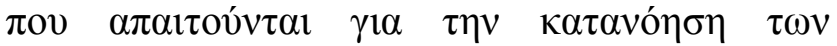

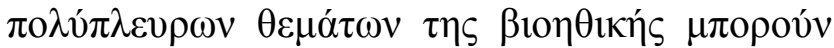

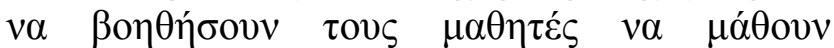

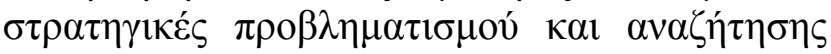

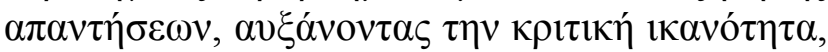

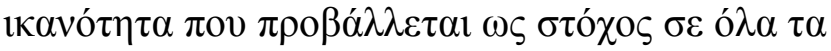

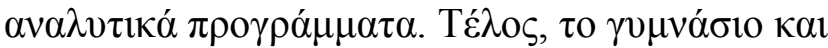

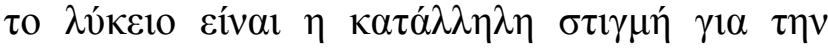

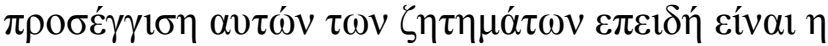

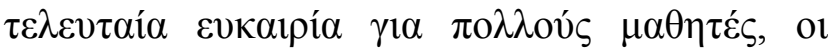

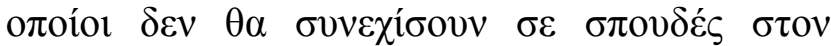

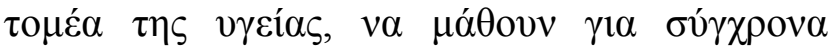

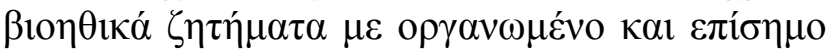

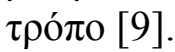

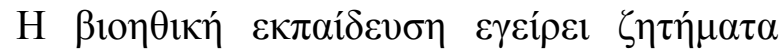

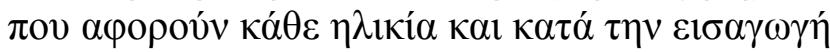

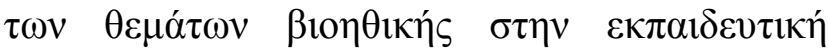

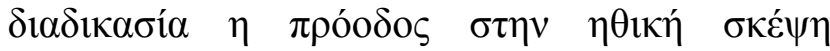

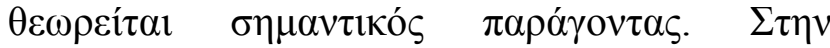

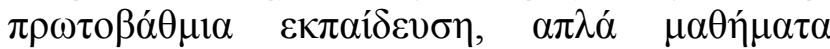

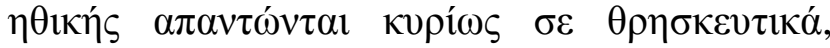

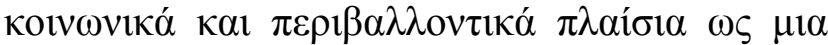

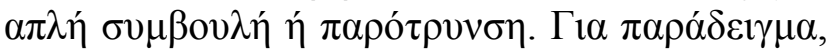

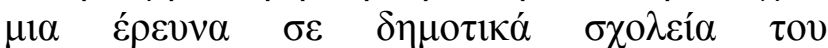

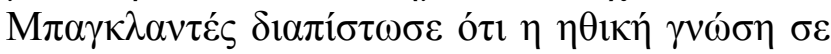

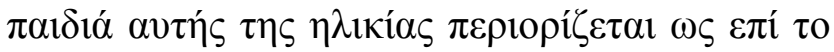

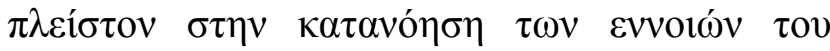

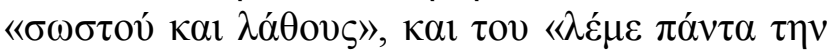

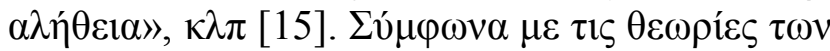

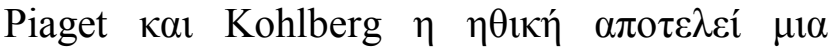

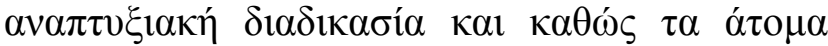

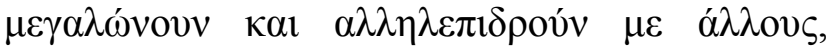

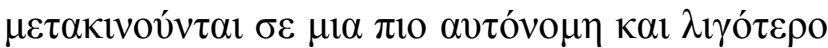

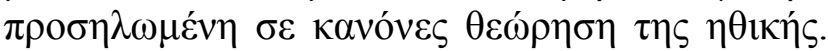

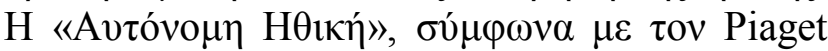

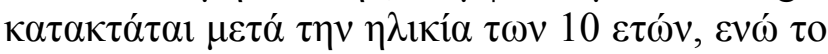

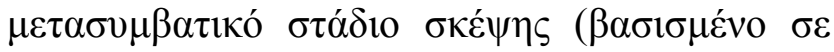

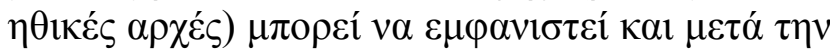

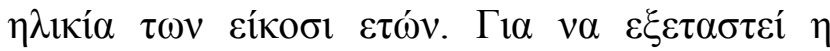

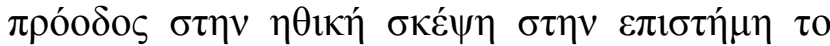

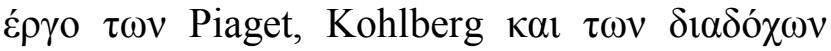

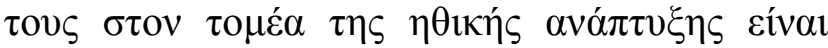

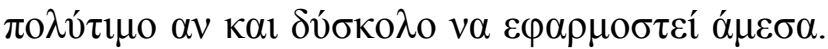

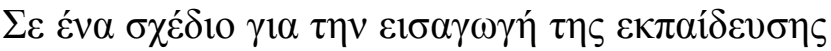

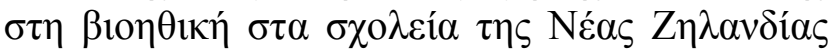

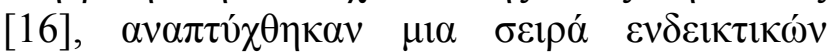

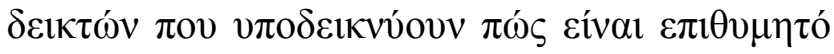

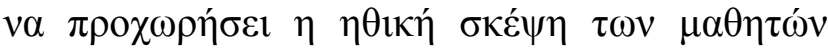
(Eıкóva 1). 

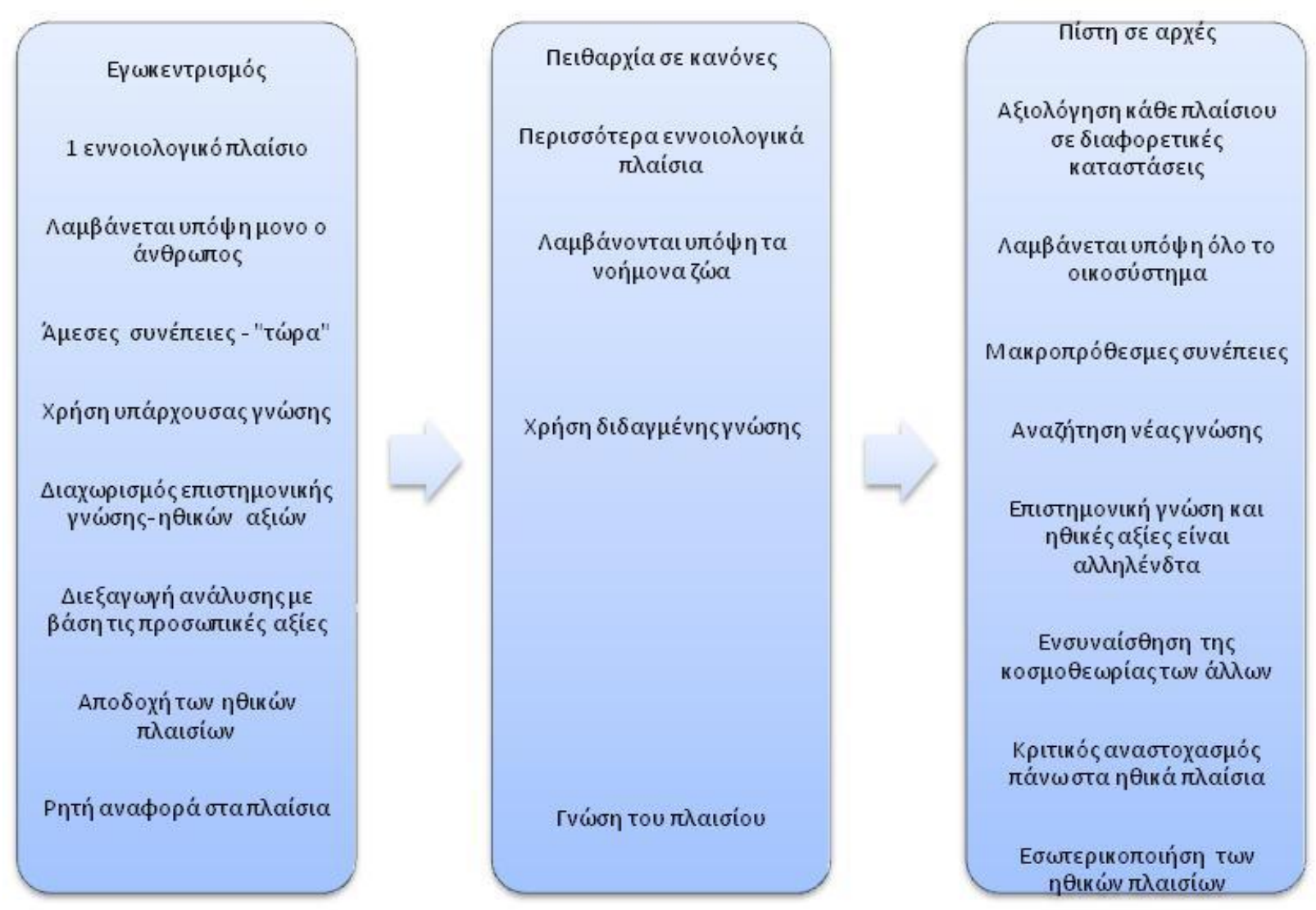

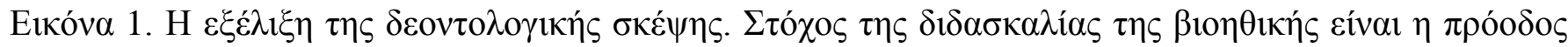

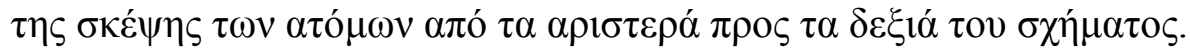




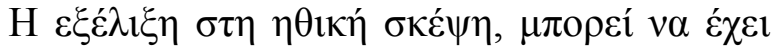

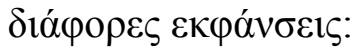

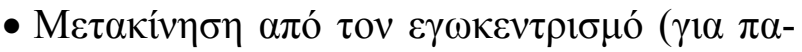

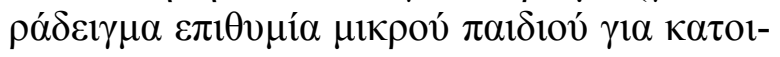

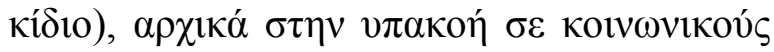

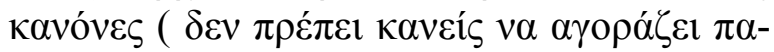

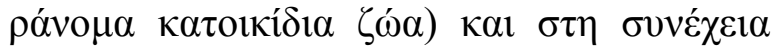
$\sigma \tau \eta v \tau \eta ́ \rho \eta \sigma \eta ~ \alpha \imath \tau \imath o \lambda o \gamma \eta \mu \varepsilon ́ v \omega v ~ \alpha \rho \chi \omega ́ v ~(\gamma \imath \alpha \pi \alpha-$

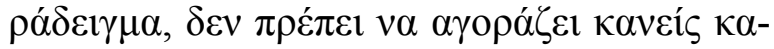

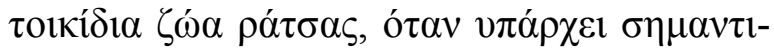

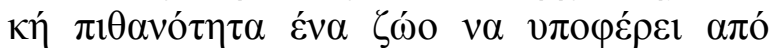

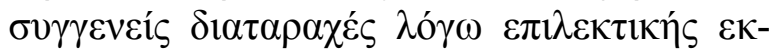

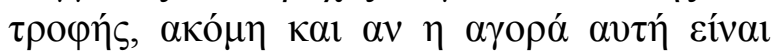
vó $\mu \mu \eta)$.

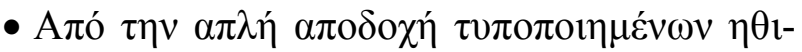

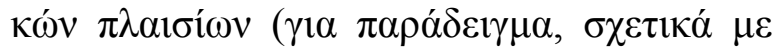

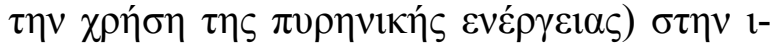

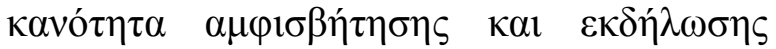

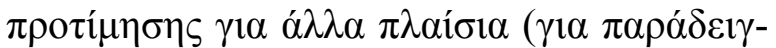

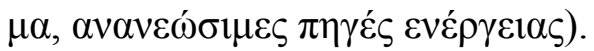

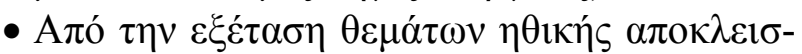

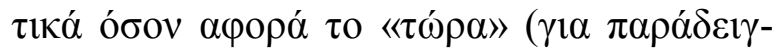

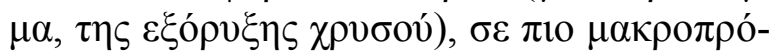

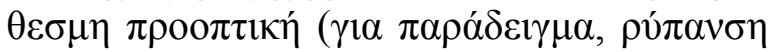

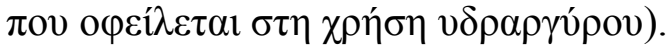

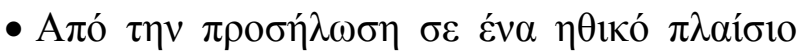

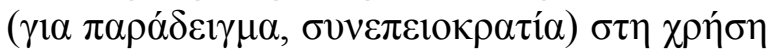

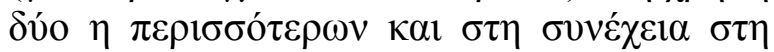

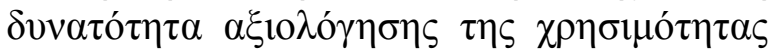

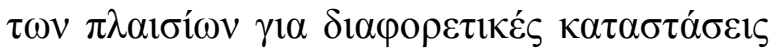

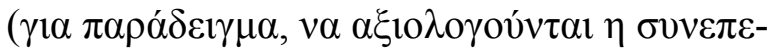

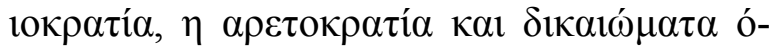

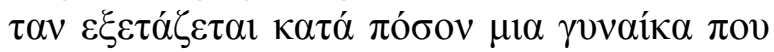

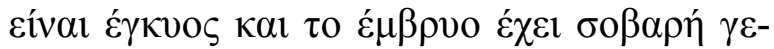

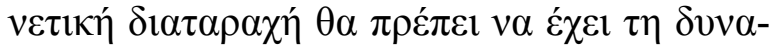

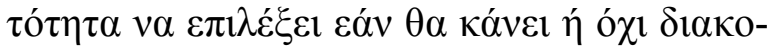

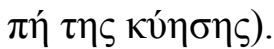

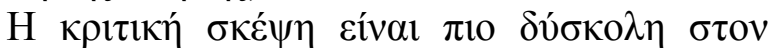

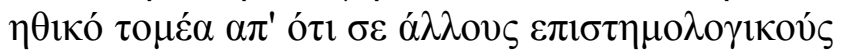

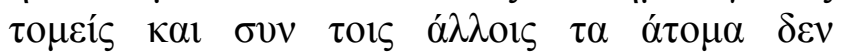

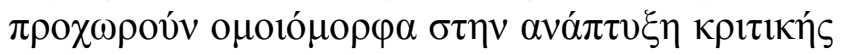

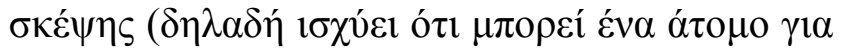

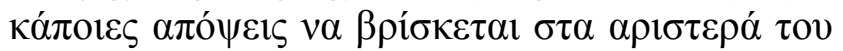

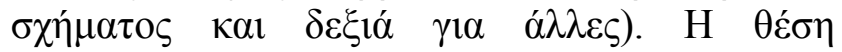

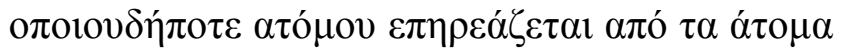

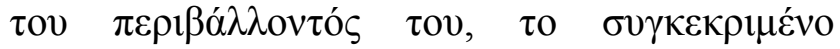

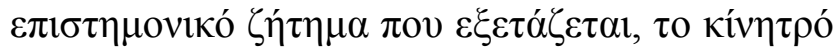

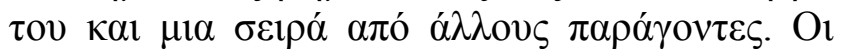

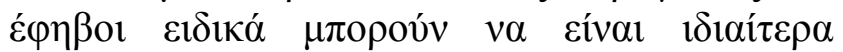

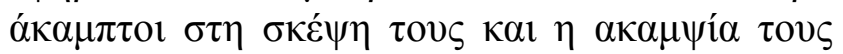

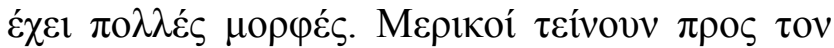

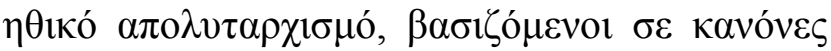

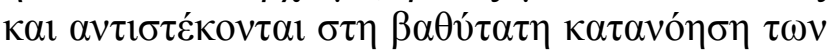

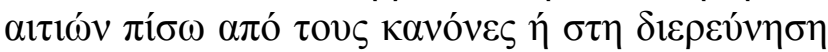

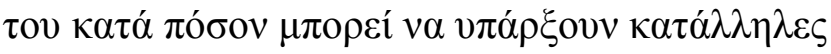

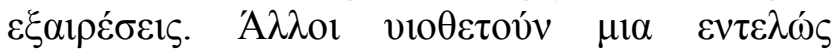

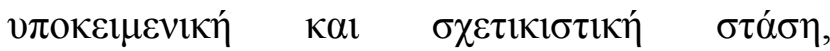

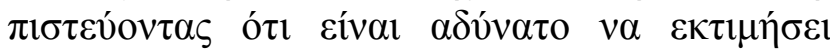

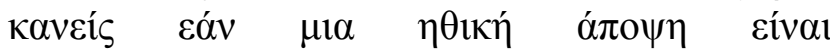

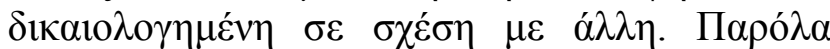

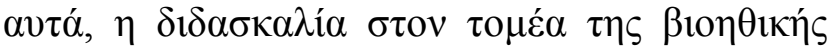

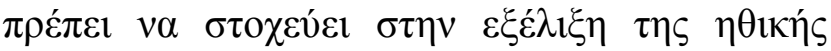

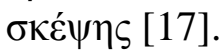

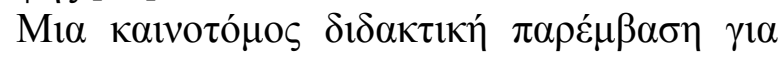

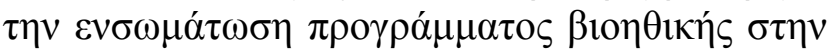
$\pi \rho \omega \tau о \beta \alpha ́ \theta \mu 1 \alpha \quad \varepsilon \kappa \pi \alpha i ́ \delta \varepsilon v \sigma \eta \quad \varepsilon \varphi \alpha \rho \mu o ́ \sigma \tau \eta \kappa \varepsilon \quad \sigma \tau \eta$

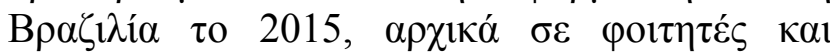

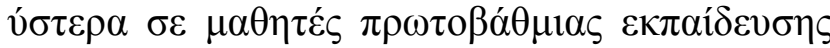

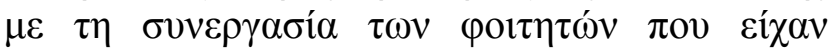

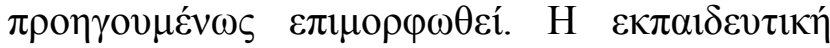

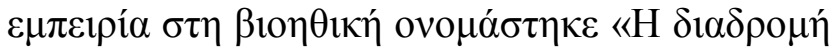

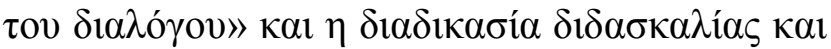

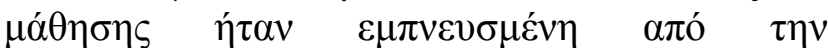

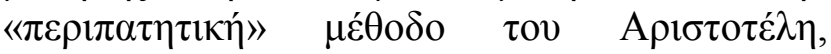

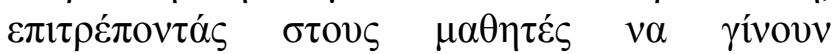

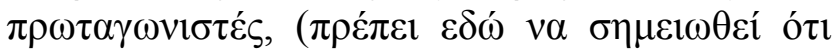

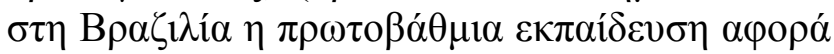

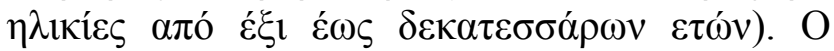

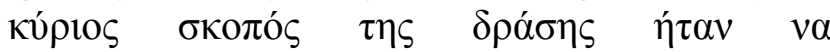

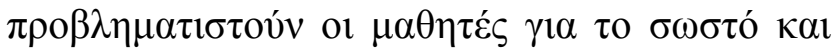

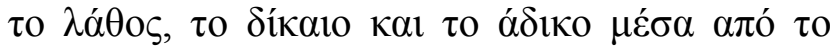

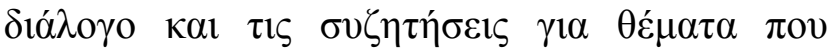

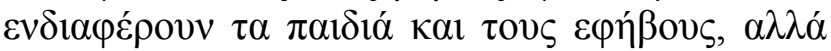

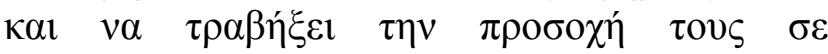

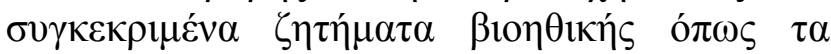

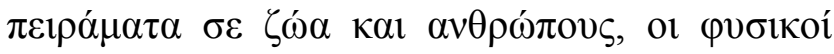

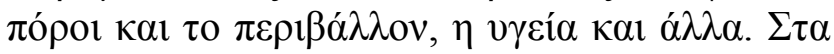

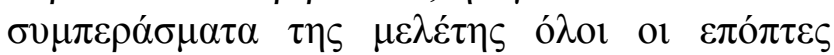

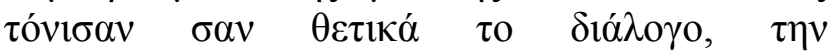

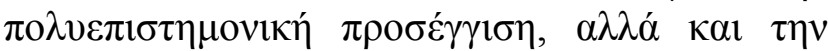

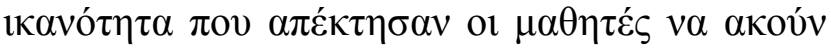

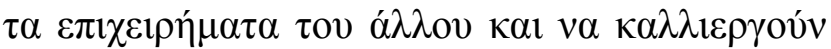

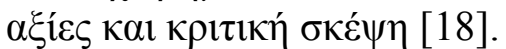

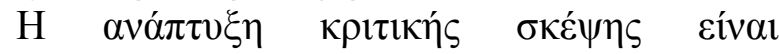

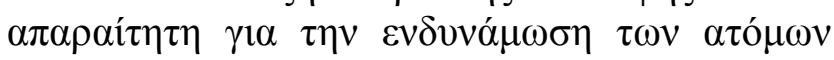

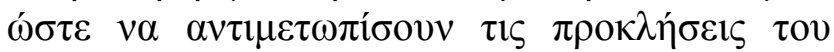

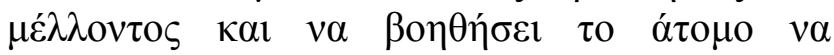

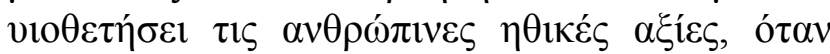

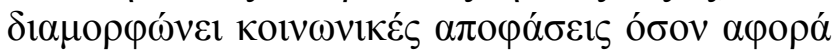

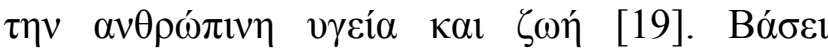




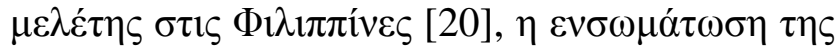

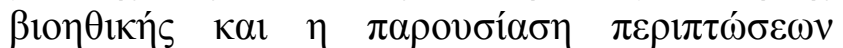

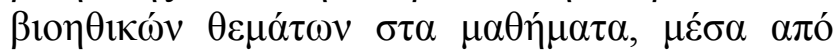

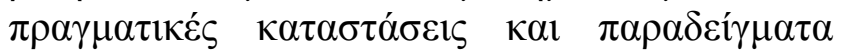

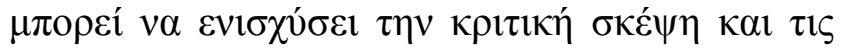

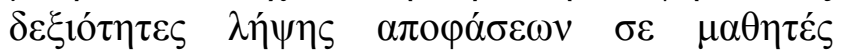

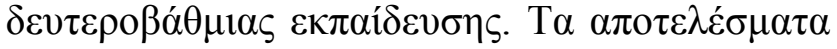

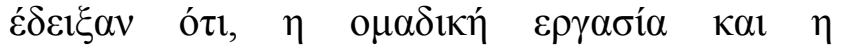

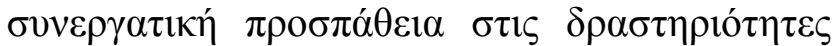

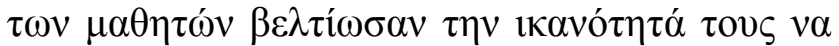

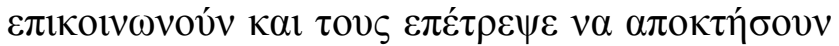

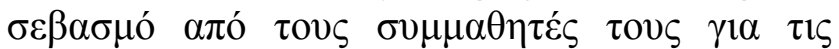

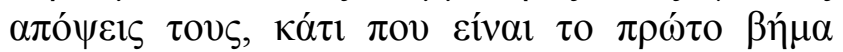

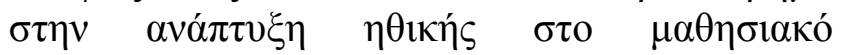
$\pi \varepsilon \rho 1 \beta \alpha ́ \lambda \lambda \mathrm{ov}$.

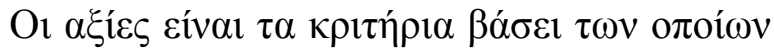

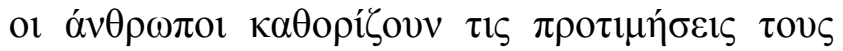

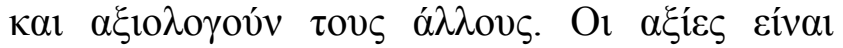

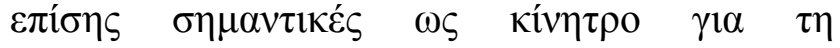

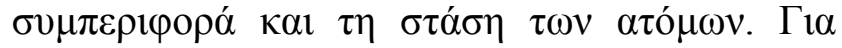

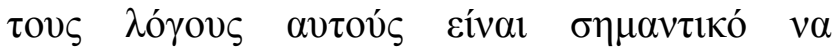
$\gamma v \omega \rho i ́ \zeta o v \mu \varepsilon \pi \omega ́ \varsigma \kappa \alpha 1 \mu \varepsilon \pi 01 \varepsilon \varsigma \alpha \xi i ́ \varepsilon \varsigma$ or $\mu \alpha \theta \eta \tau \dot{\varepsilon} \varsigma$

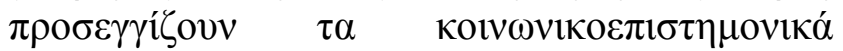

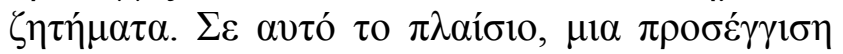

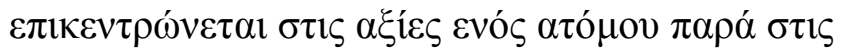

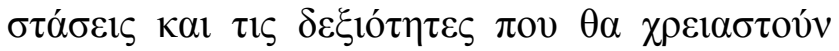

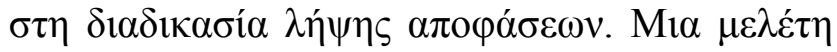

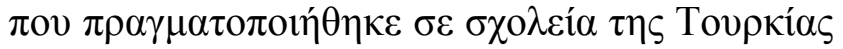

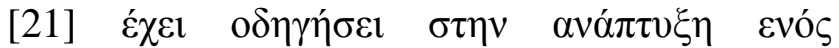

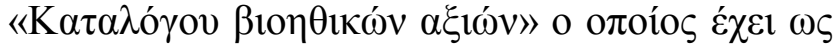

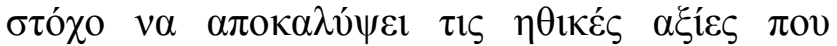

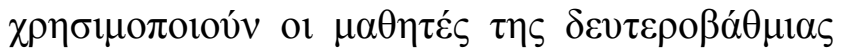

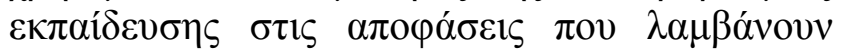

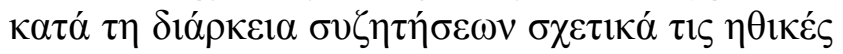

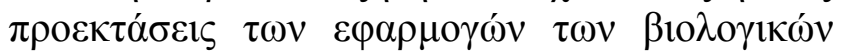

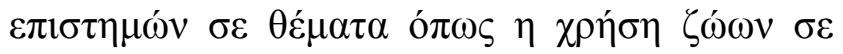

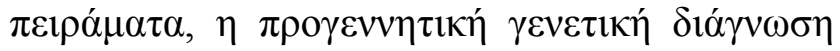

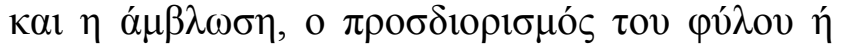

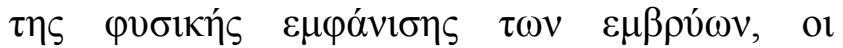

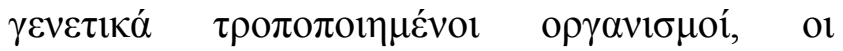

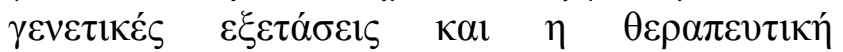

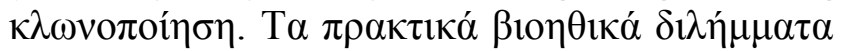

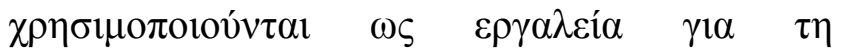

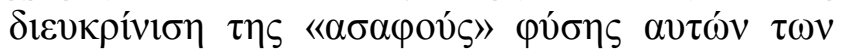

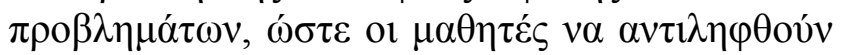

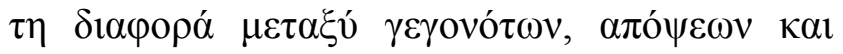
$\alpha \xi ı \dot{v}$.

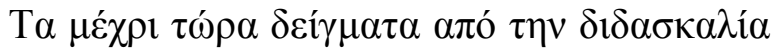

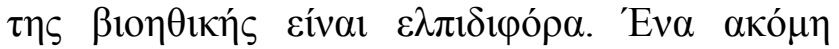

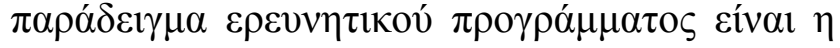

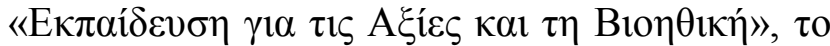
олоío $\alpha \pi \varepsilon v \theta v ́ v \theta \eta \kappa \varepsilon \quad \sigma \varepsilon \quad \mu \alpha \theta \eta \tau \dot{\varepsilon} \varsigma \quad \delta \eta \mu o ́ \sigma 1 \alpha \varsigma$

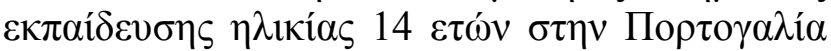

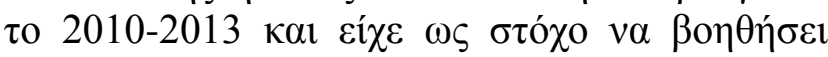

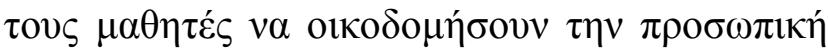

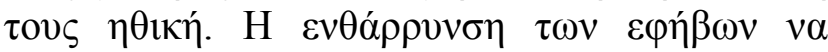

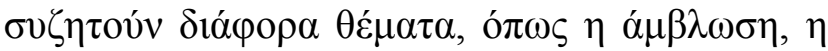

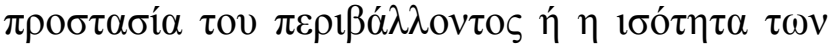

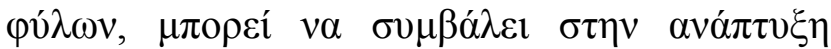

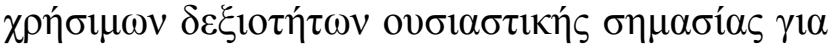

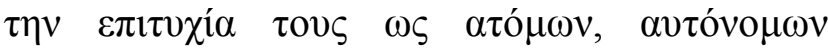

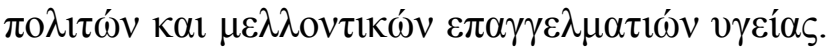

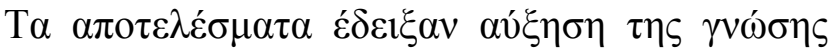

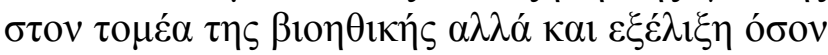

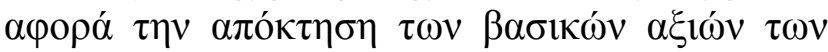

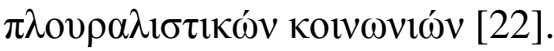

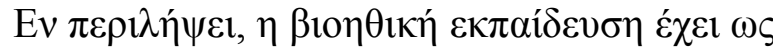

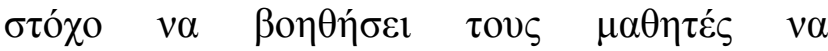

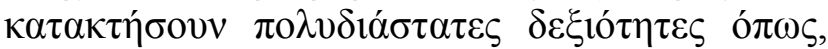

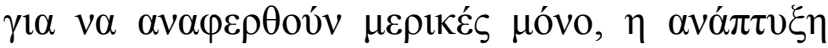

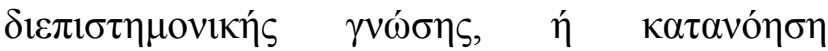

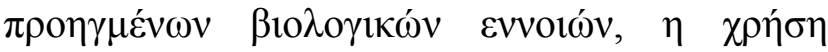

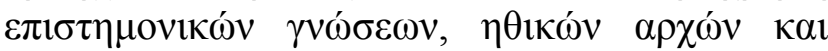

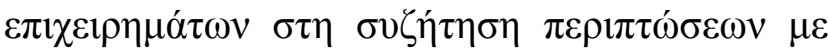

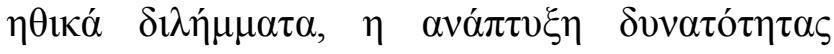

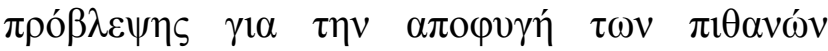

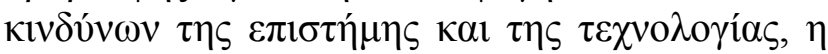

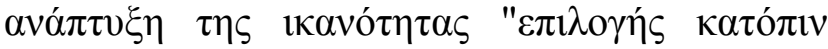

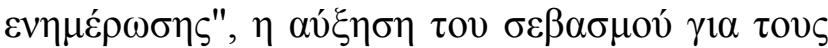

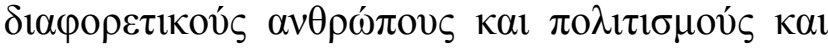

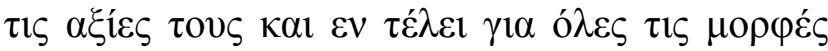

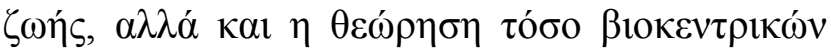

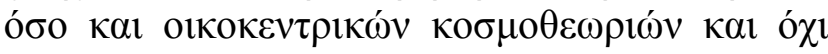

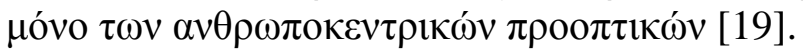

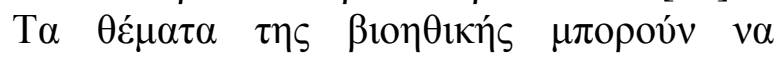

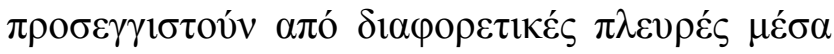

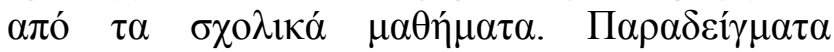

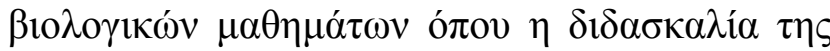

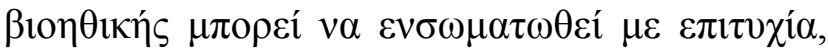

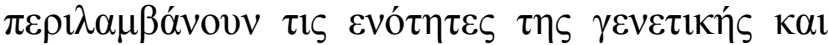

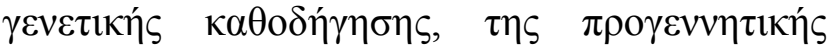

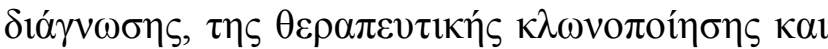

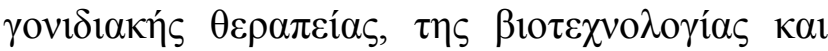

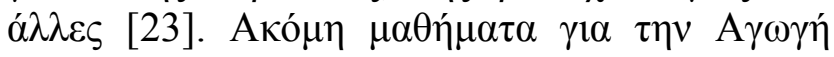

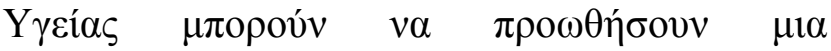

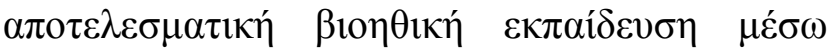
$\theta \varepsilon \mu \alpha \tau o \lambda o \gamma i \alpha \varsigma$ or $\mu \varepsilon \tau \alpha \mu \circ \sigma \chi \varepsilon v ́ \sigma \varepsilon 1 \varsigma, \eta$ in vitro

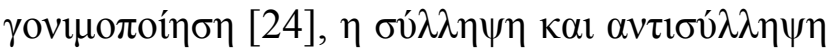

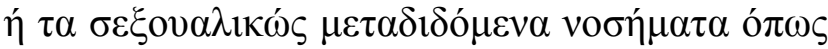

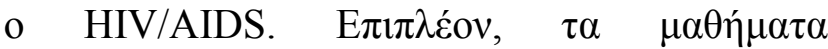




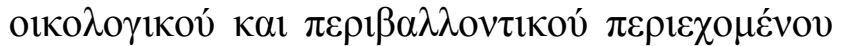

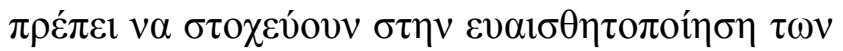

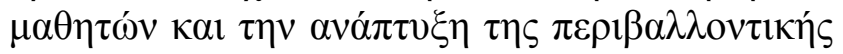

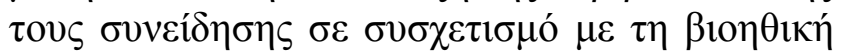

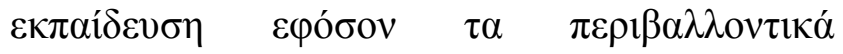

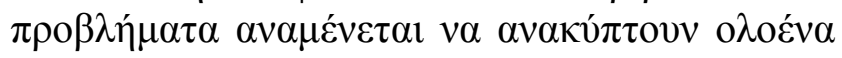

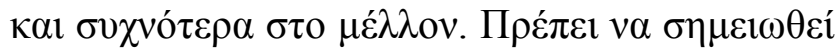

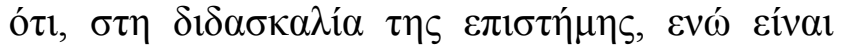

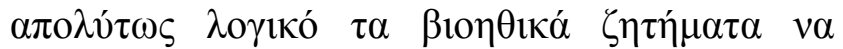

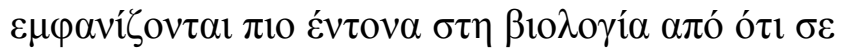

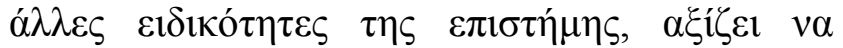

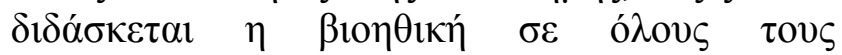

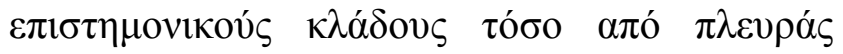

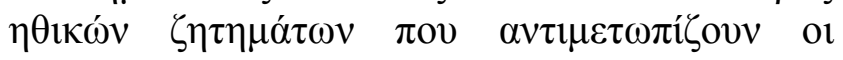
$\varepsilon \pi 1 \sigma \tau \eta \dot{\mu o v \varepsilon \varsigma ~ o ́ \sigma o ~ \sigma \varepsilon ~ o ́ \tau ı ~ \alpha \varphi о \rho \alpha ́ ~ \tau ı \zeta ~ \varepsilon \varphi \alpha \rho \mu о \gamma \varepsilon ́ \varsigma ~ \tau \eta ऽ ~}$

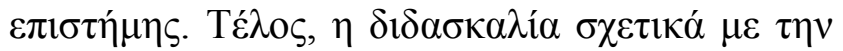

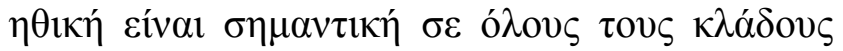

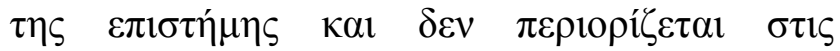

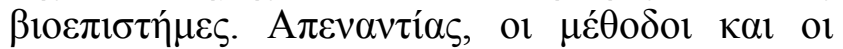

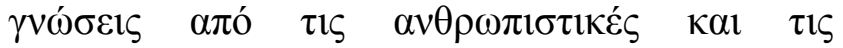

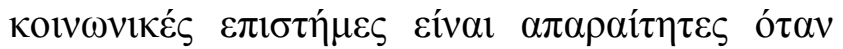
$\varepsilon \pi 1 \delta 1 \omega ́ \kappa \varepsilon \tau \alpha \imath \quad \eta \quad \alpha v \tau 1 \mu \varepsilon \tau \dot{\pi} \pi 1 \sigma \eta \quad \beta 10 \eta \theta 1 \kappa \omega ́ v$ $\pi \rho \circ \beta \lambda \eta \mu \alpha \tau 1 \sigma \mu \omega ́ v ~[9]$.

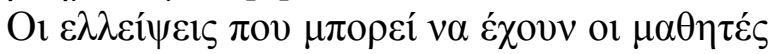

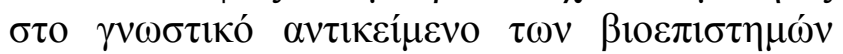

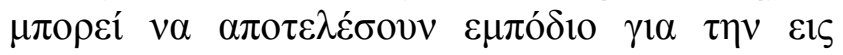

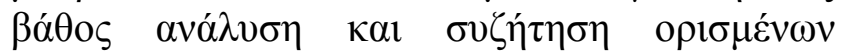

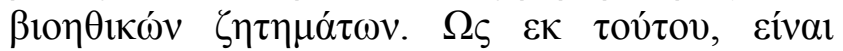

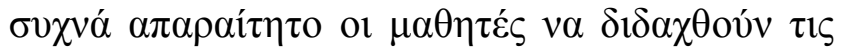

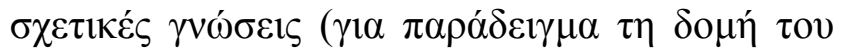

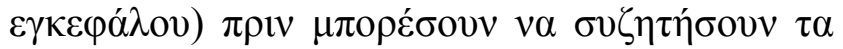

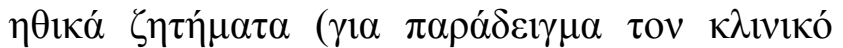

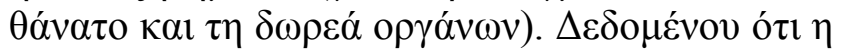

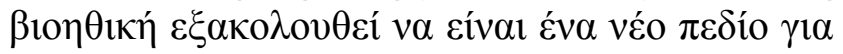

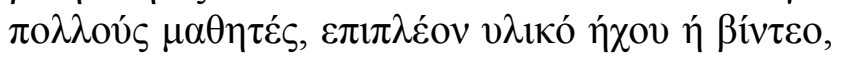

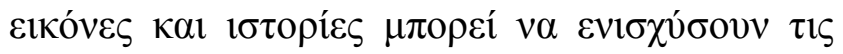

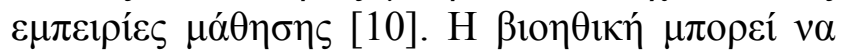
$\varepsilon 1 \sigma \varepsilon ́ \lambda \theta \varepsilon 1 \quad \sigma \varepsilon \quad \pi \mathrm{o} \lambda v \alpha \dot{\rho} \rho 1 \theta \mu \alpha \quad \mu \alpha \theta \eta \dot{\eta} \mu \alpha \tau \alpha \quad \tau \mathrm{ov}$

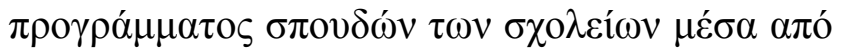

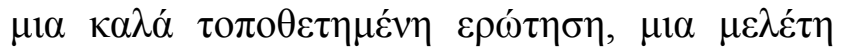

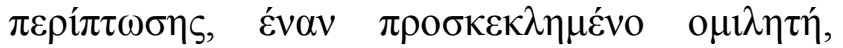

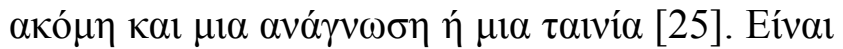

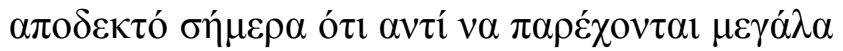

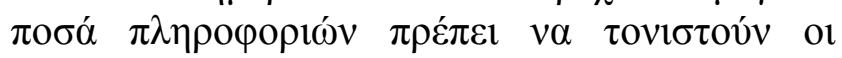

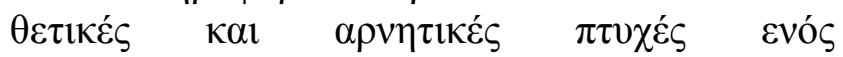

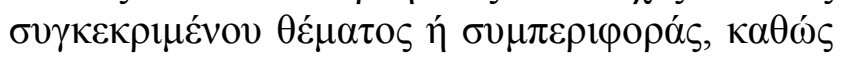

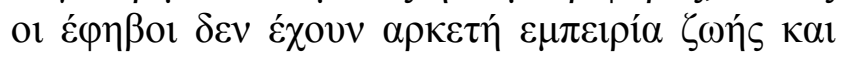

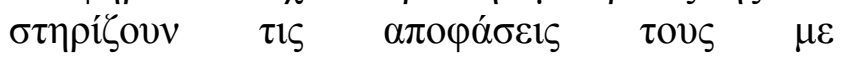

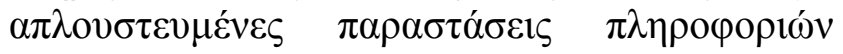
[26].

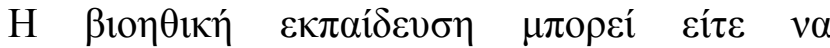

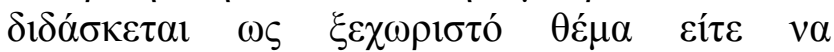

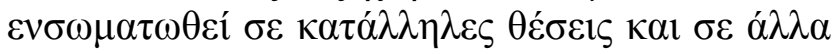

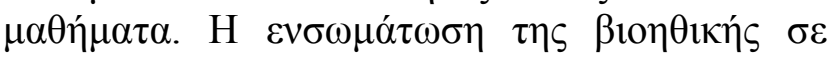

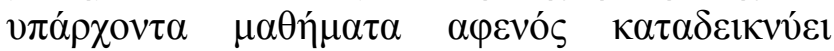

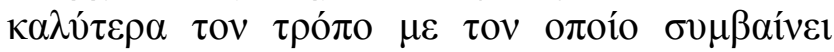

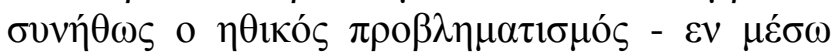

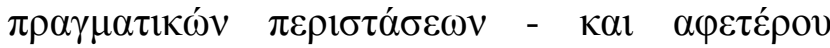

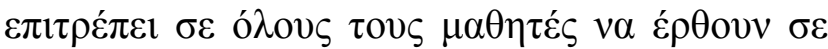

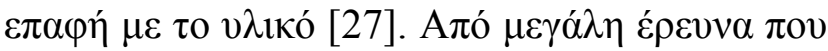

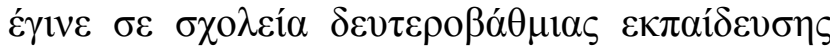

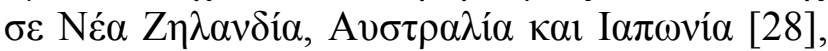

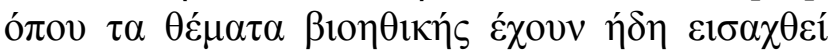

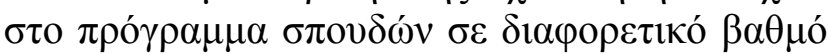

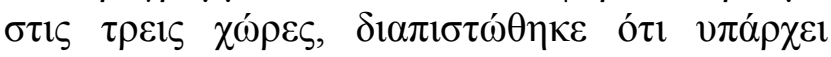

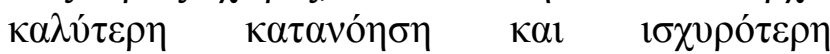

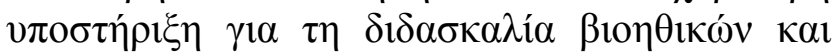

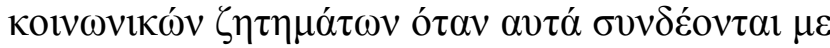

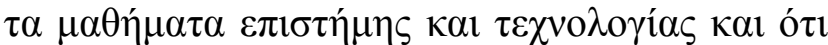

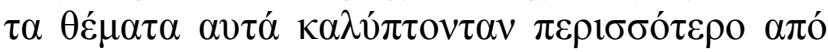

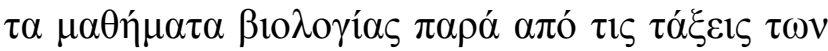

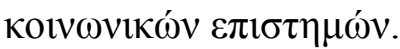

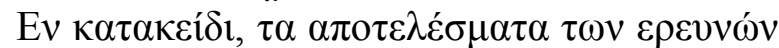

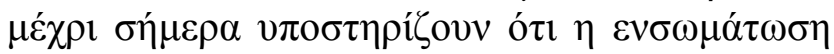

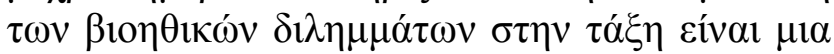

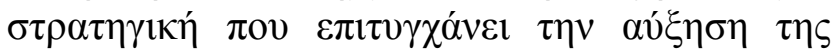

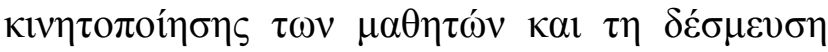

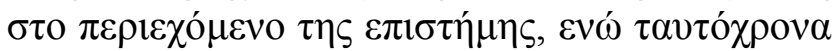

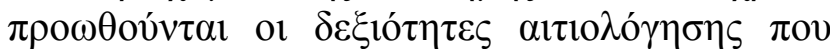

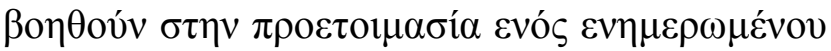

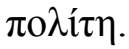

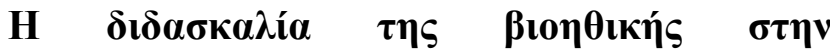

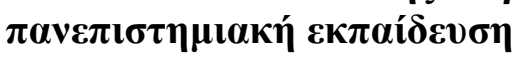

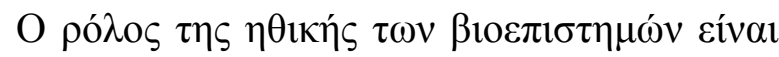

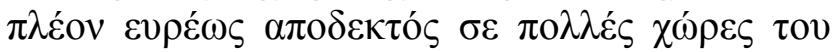

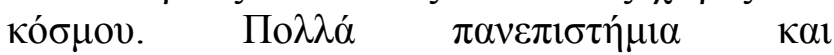

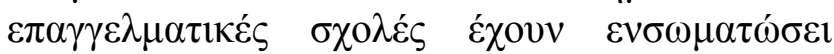

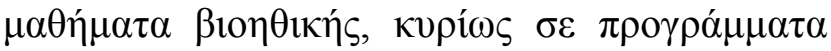

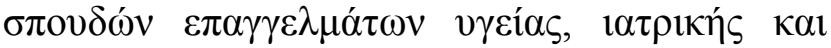

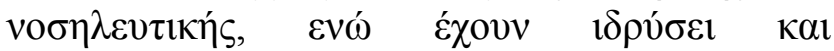

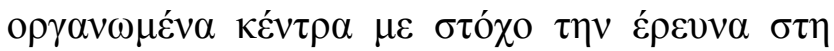

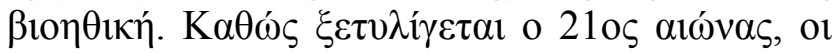

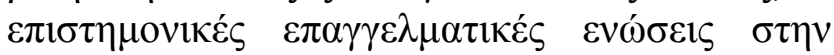

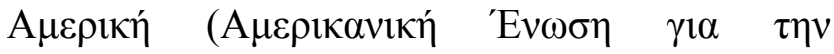

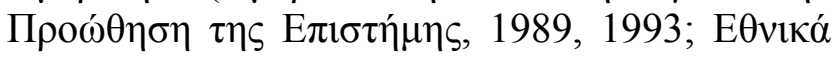

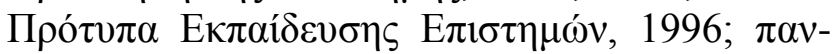

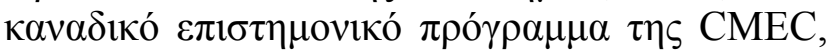

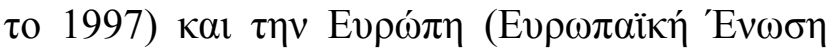




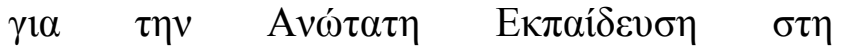

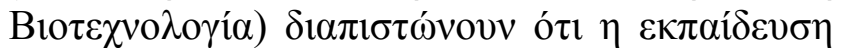

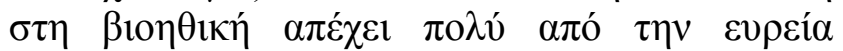

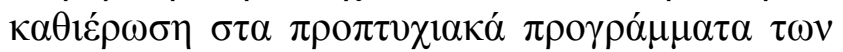

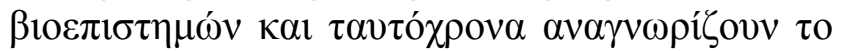

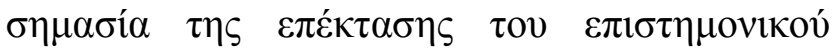

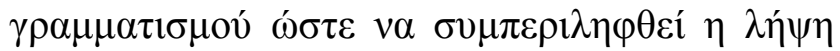
$\alpha \pi \circ \varphi \alpha ́ \sigma \varepsilon \omega \nu \quad \mu \varepsilon \quad \tau \varepsilon \kappa \mu \eta \rho \iota \omega \mu \varepsilon ́ v \eta \quad \gamma \nu \omega ́ \sigma \eta, \quad \eta$

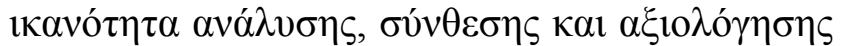
$\pi \lambda \eta \rho о \varphi о \rho і \omega ́ v ~ \sigma \varepsilon \quad \pi \varepsilon \rho 1 \sigma \sigma o ́ \tau \varepsilon \rho \alpha \quad \pi \rho о \gamma \rho \alpha ́ \mu \mu \alpha \tau \alpha$

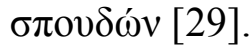

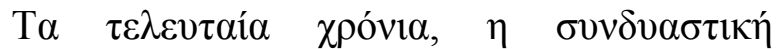

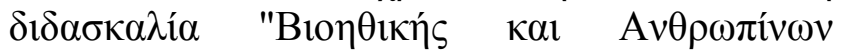

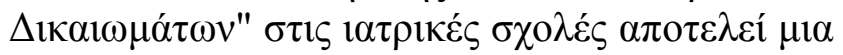

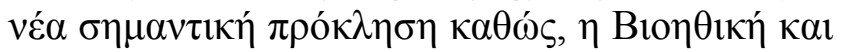

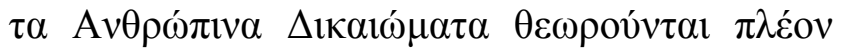

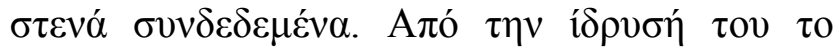

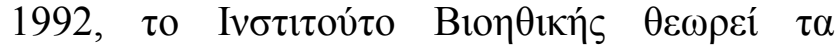

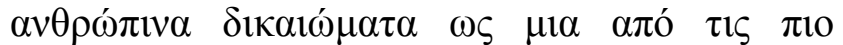

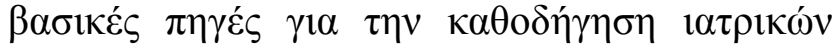

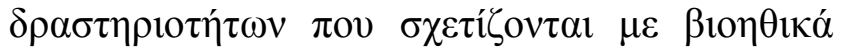

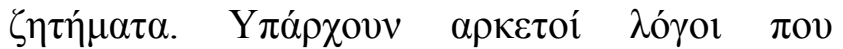

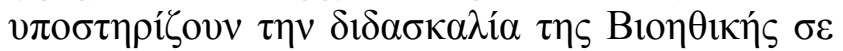

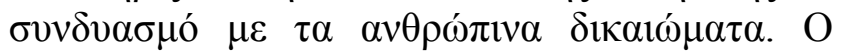

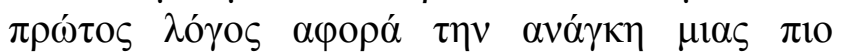

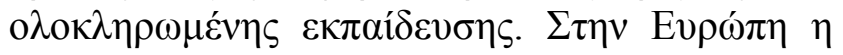

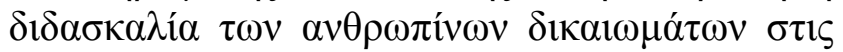

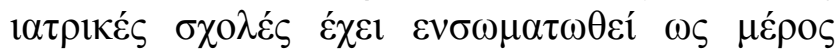

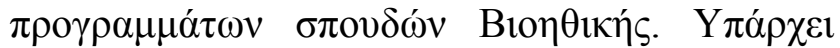

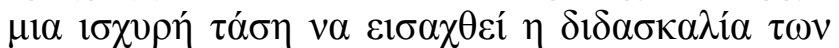

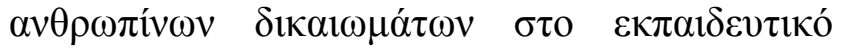

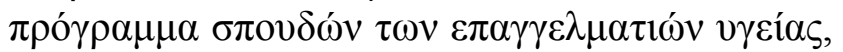

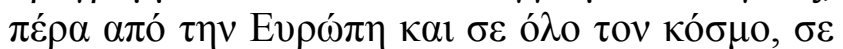

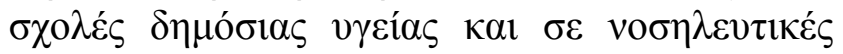

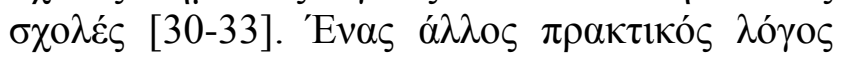

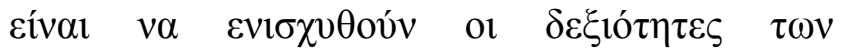

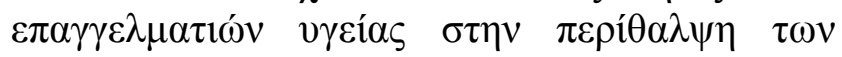

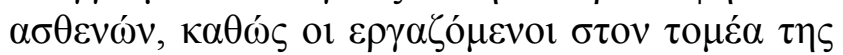

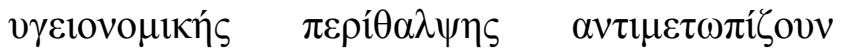

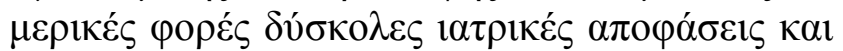

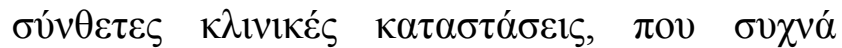

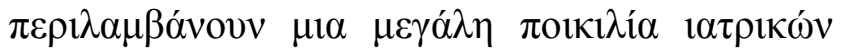

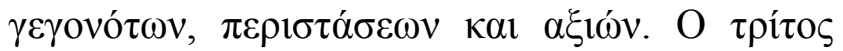

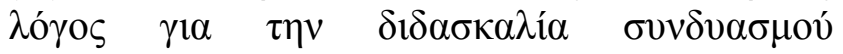

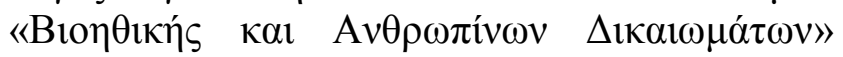

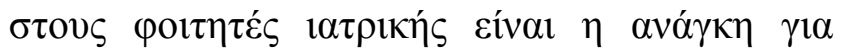

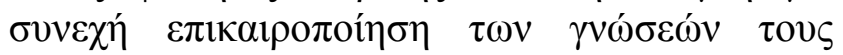

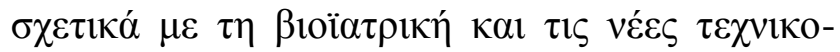

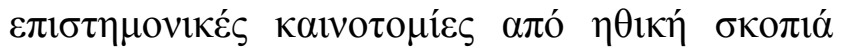
[34-35].

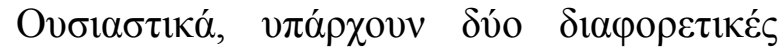

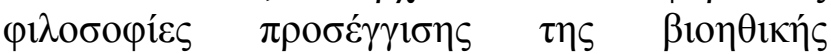

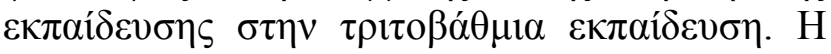
$\mu 1 \alpha \quad \pi \rho \circ \sigma \varepsilon ́ \gamma \gamma 1 \sigma \eta, \quad \pi 10 \quad \pi \varepsilon \rho 10 \rho 1 \sigma \mu \varepsilon ́ v \eta \quad \kappa \alpha 1$

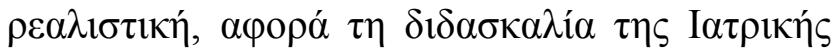

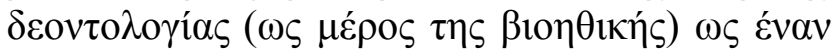

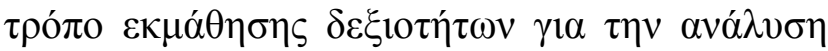

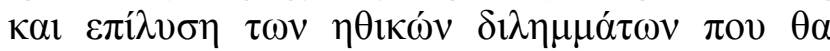

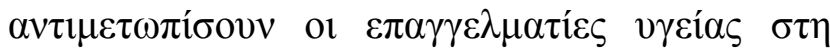

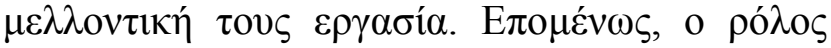

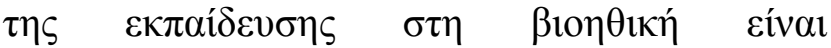

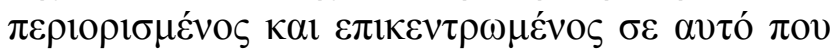

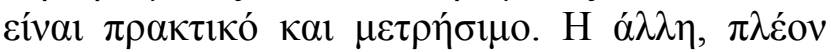

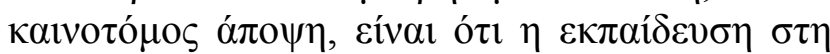

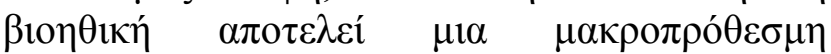

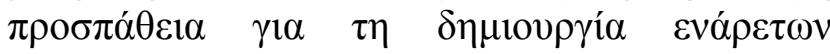

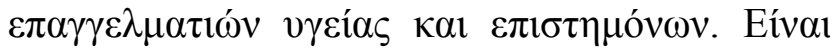

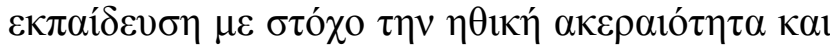

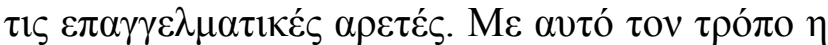

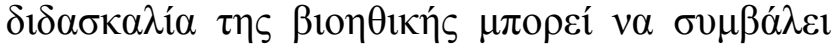

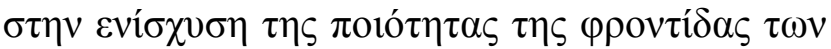

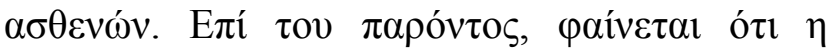

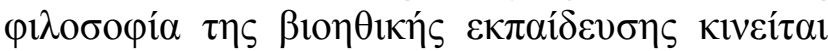

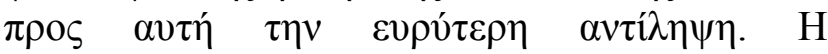

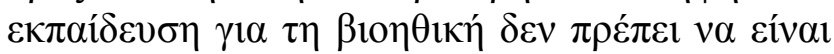

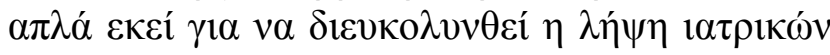

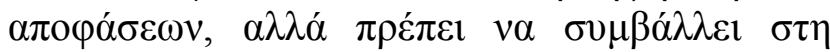

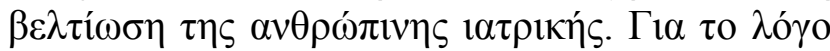

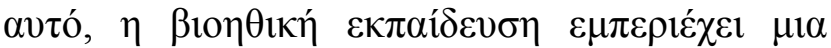

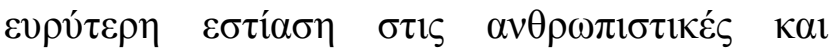

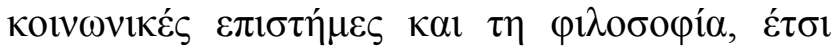

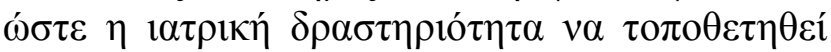

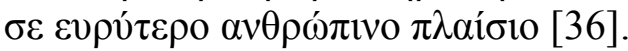

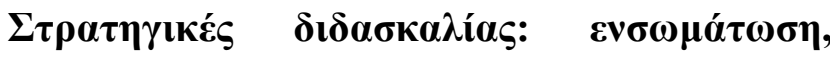

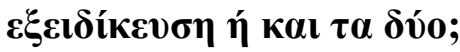

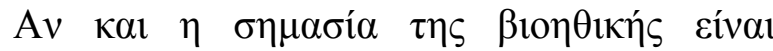

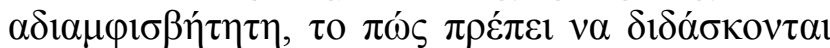

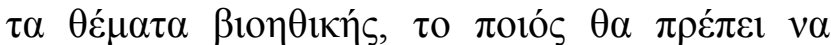

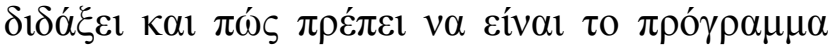

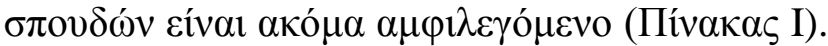

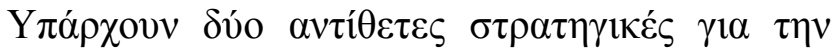

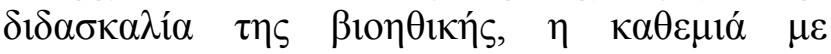

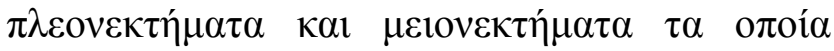

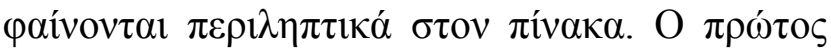

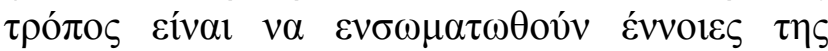

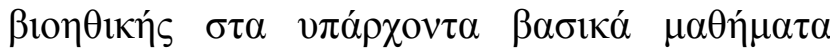

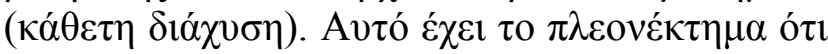

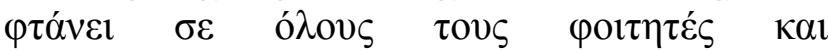


$\varepsilon \kappa \pi \alpha 1 \delta \varepsilon v o ́ \mu \varepsilon v o v \varsigma \quad \alpha \lambda \lambda \alpha \dot{\alpha}, \quad \sigma \varepsilon \quad \varepsilon \dot{v} \alpha \alpha \quad \gamma \varepsilon \mu \alpha \dot{\tau} \mathrm{o}$

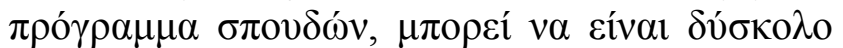

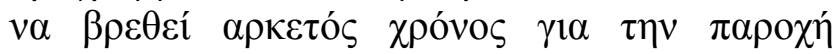

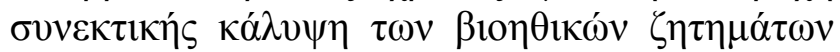

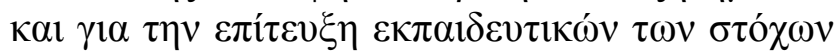

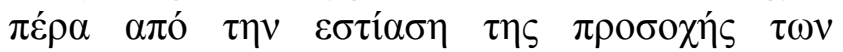

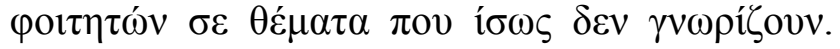

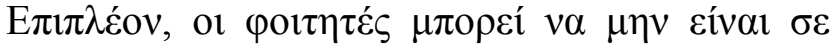

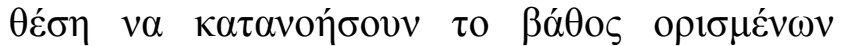

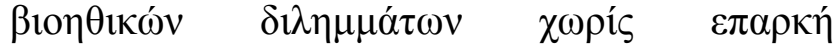

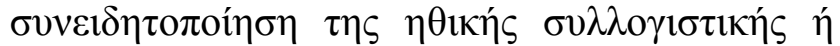

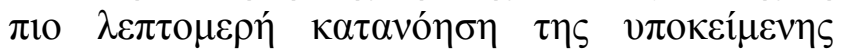

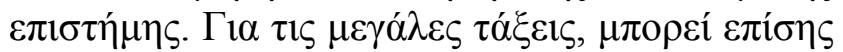

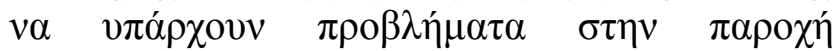

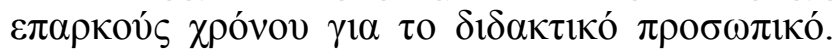

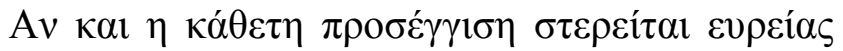

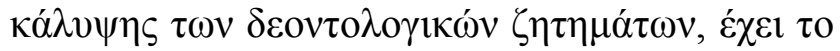

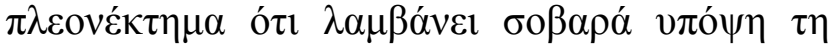

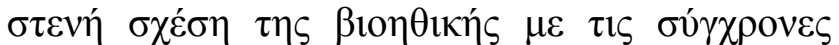

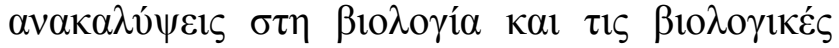
$\alpha \rho \chi \varepsilon ́ \varsigma[37,38]$.

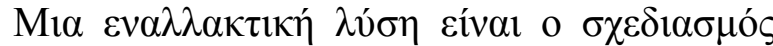

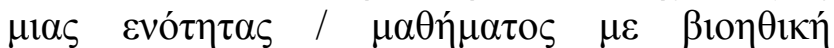

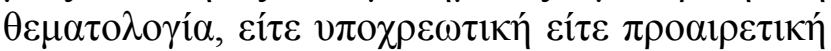

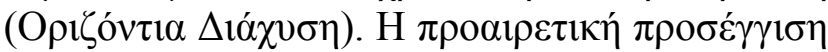

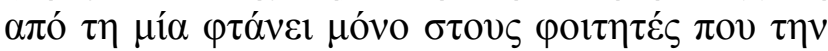

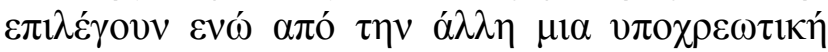

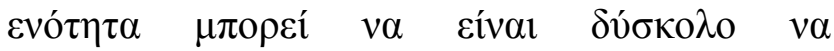

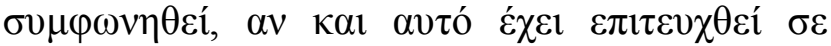

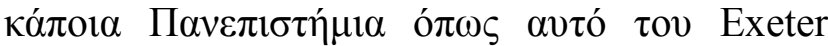

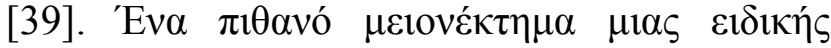

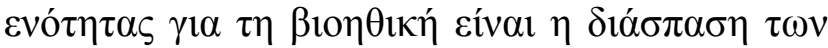

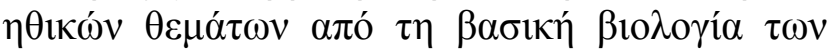

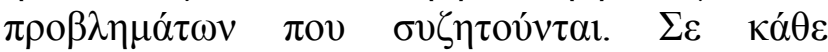
$\pi \varepsilon \rho i ́ \pi \tau \omega \sigma \eta, \quad \eta \quad \pi \alpha \rho o v \sigma i ́ \alpha \sigma \eta \quad \tau \omega v \quad \theta \varepsilon \mu \alpha ́ \tau \omega \nu$

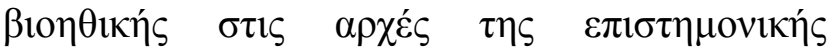

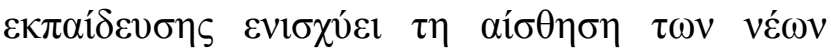

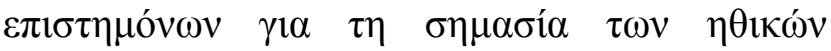
$\pi \rho \circ \beta \lambda \eta \mu \alpha \tau \imath \sigma \mu \omega ́ v \quad \sigma \tau \eta \quad \mu \varepsilon \lambda \lambda \sigma \nu \tau 1 \kappa \eta ் ~ \tau o v \varsigma$

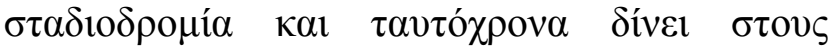

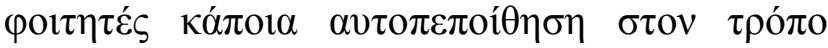

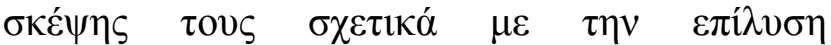

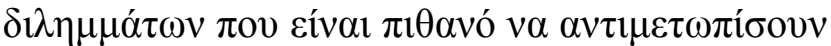
$[40,41]$ 


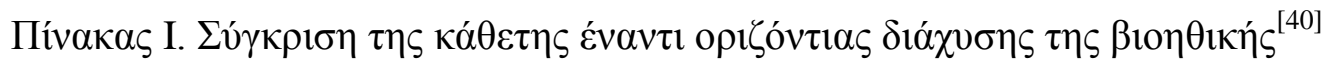

\begin{tabular}{|c|c|c|c|}
\hline \multicolumn{4}{|c|}{ 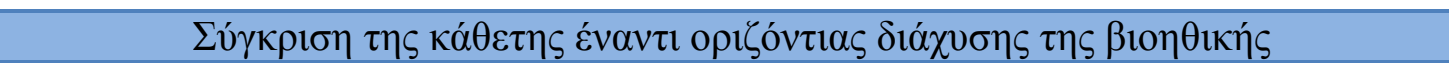 } \\
\hline \multicolumn{2}{|c|}{ 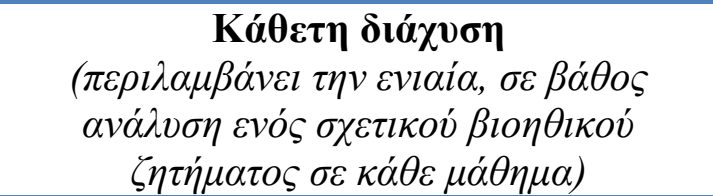 } & \multicolumn{2}{|c|}{ 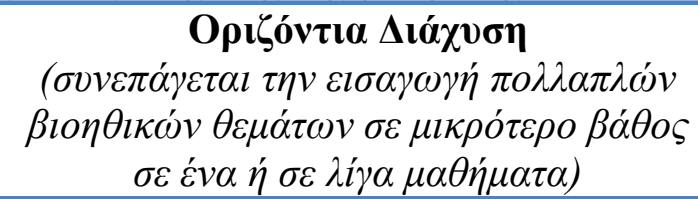 } \\
\hline Yл⿻́丿 & 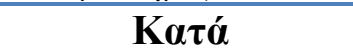 & Y $\pi \dot{\varepsilon} \rho$ & 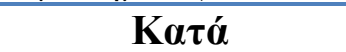 \\
\hline 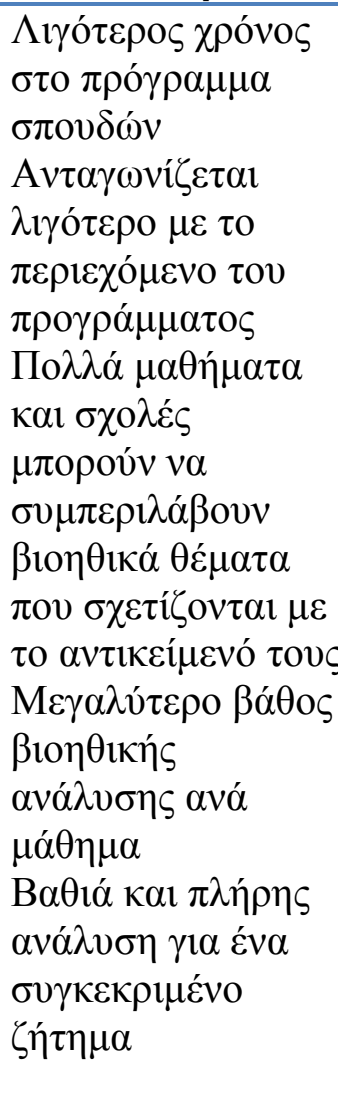 & 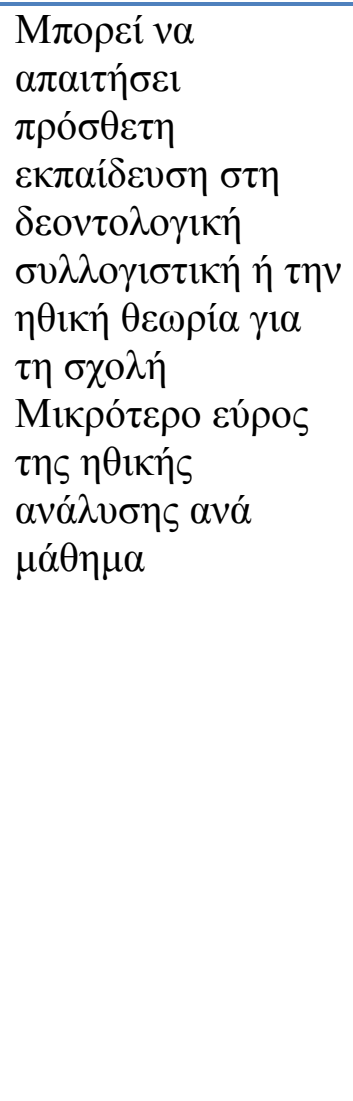 & 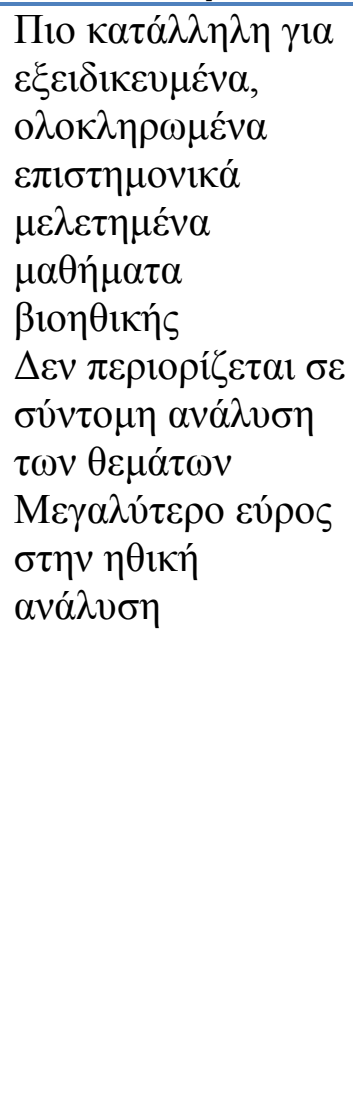 & 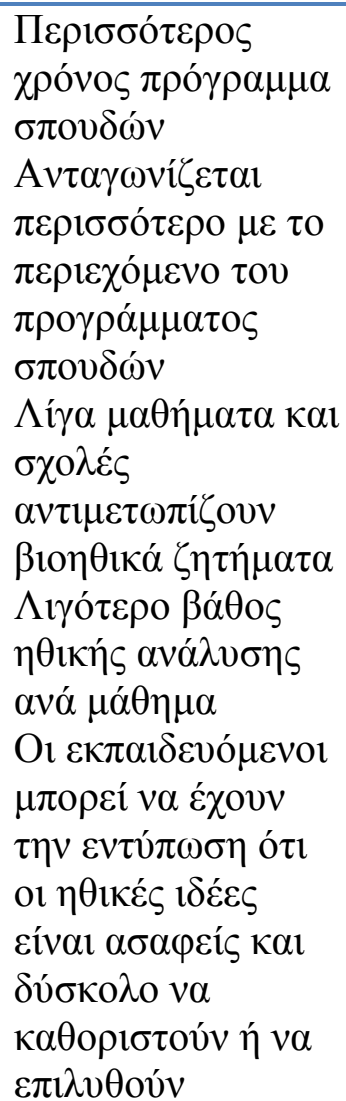 \\
\hline
\end{tabular}




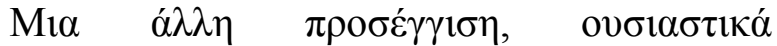

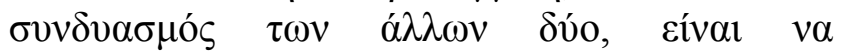

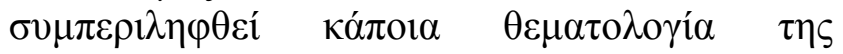

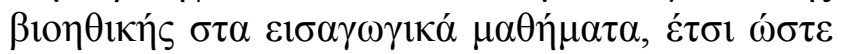

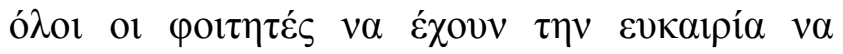

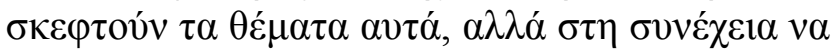

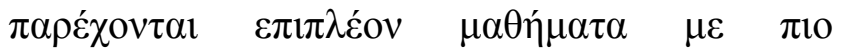

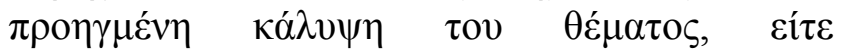

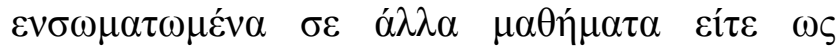

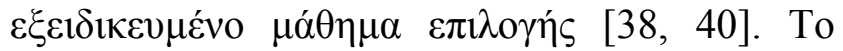

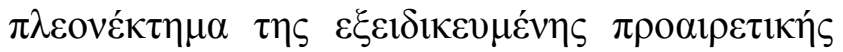

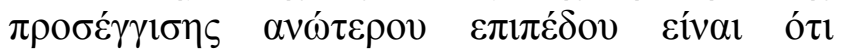

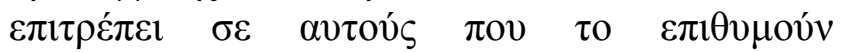

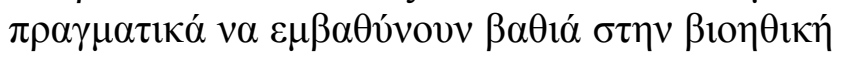
[42].

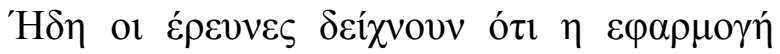

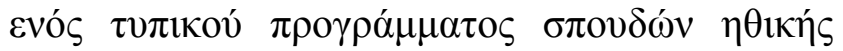

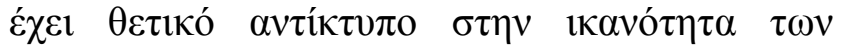

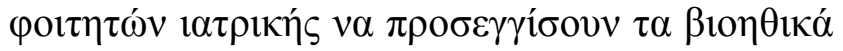

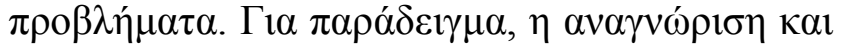

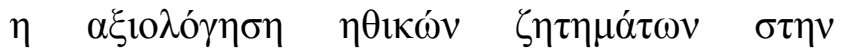

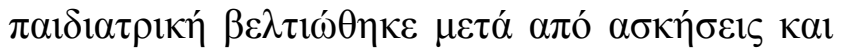

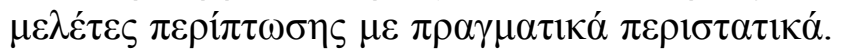

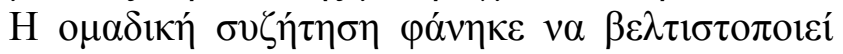

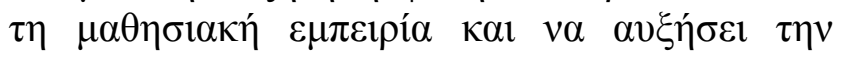

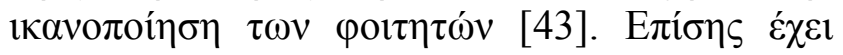

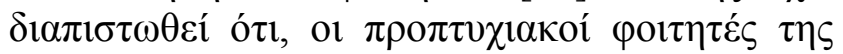

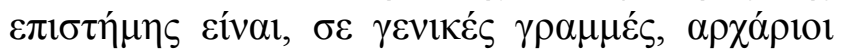

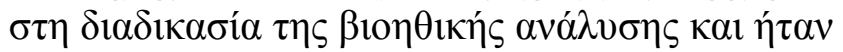

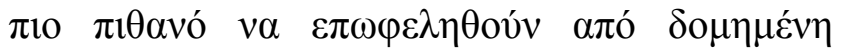

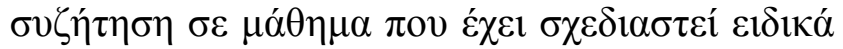

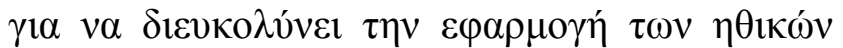

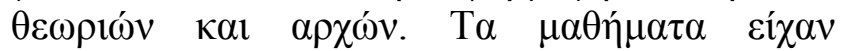

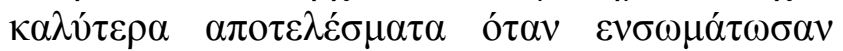

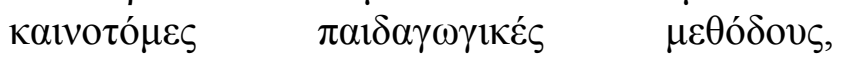
$\pi \varepsilon \rho 1 \pi \tau \omega \sigma 10 \lambda \mathrm{o} \gamma 1 \kappa \varepsilon ́ \varsigma \mu$

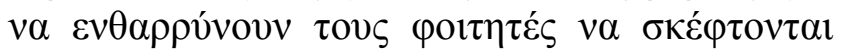

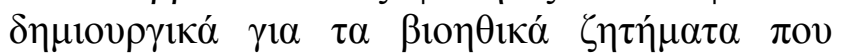

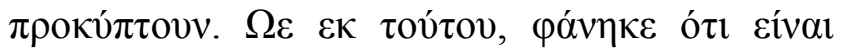

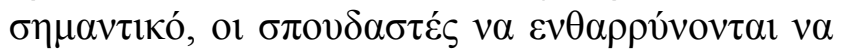

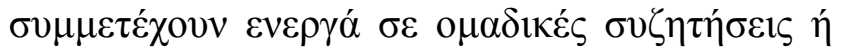

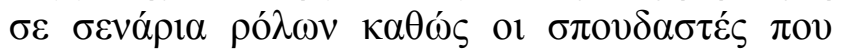

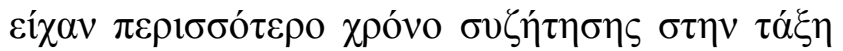

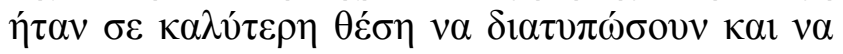

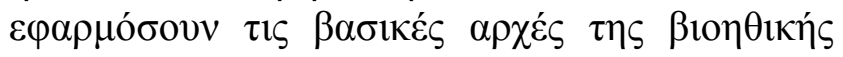

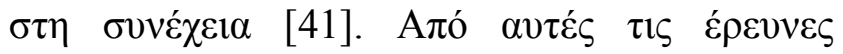

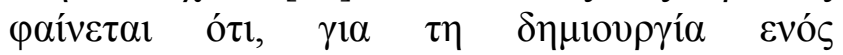

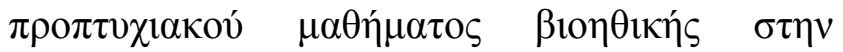

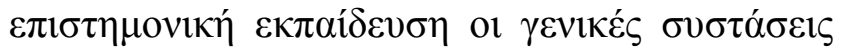

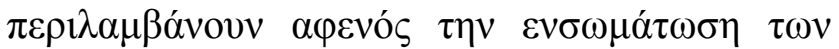

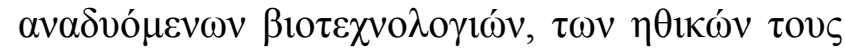

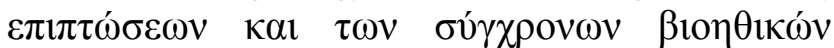

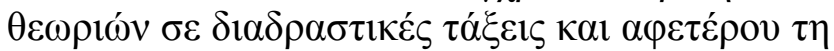
$\delta i \alpha ́ \rho \theta \rho \omega \sigma \eta \quad \tau \omega v \quad \mu \alpha \theta \eta \mu \alpha ́ \tau \omega v \quad \mu \varepsilon \quad \beta \alpha ́ \sigma \eta \quad \tau \eta$

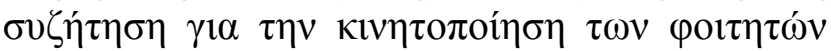

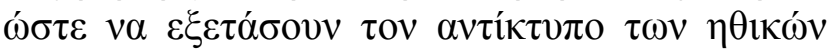

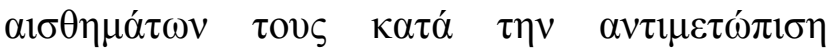

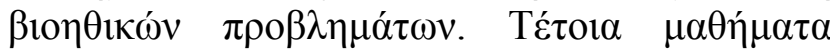
$\pi v \rho \circ \delta$ o

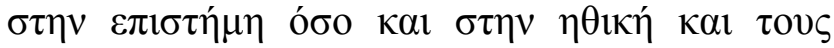

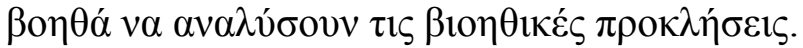

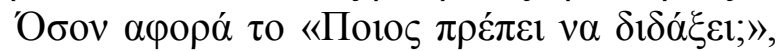

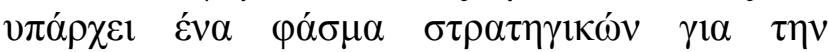

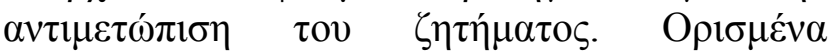

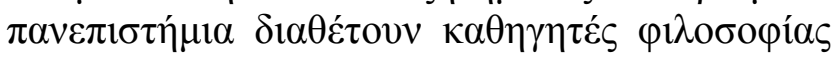
$\gamma 1 \alpha \quad v \alpha \quad \delta 1 \delta \alpha \dot{\xi}$ ovv $\tau$ ov

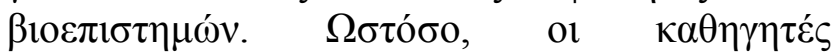

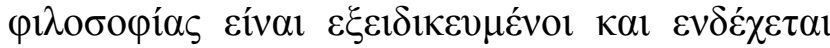

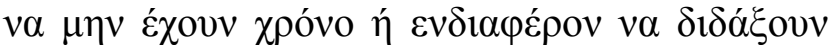

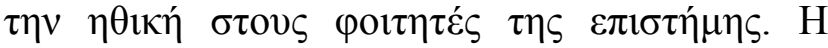

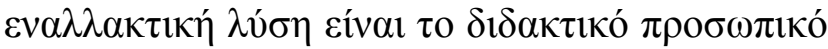

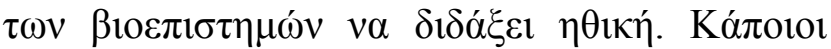

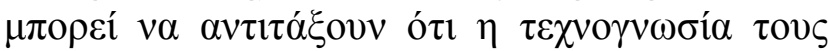

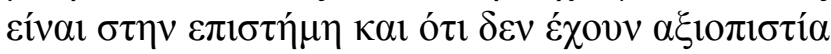

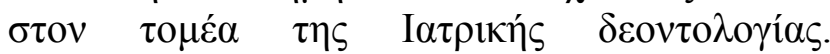

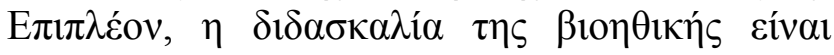

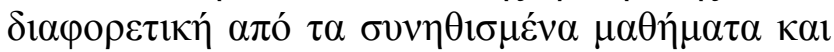

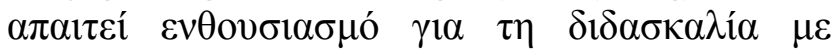

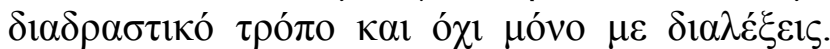

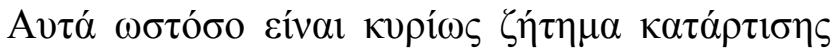

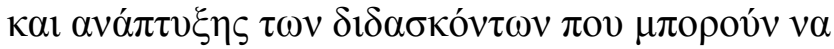

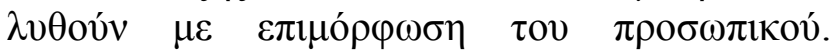

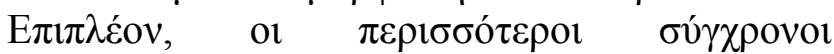

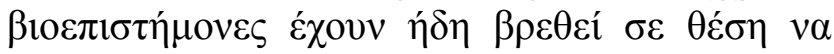

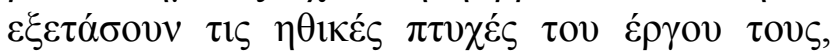

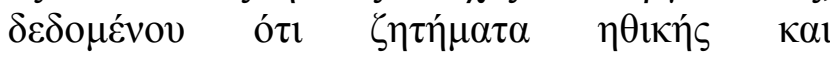

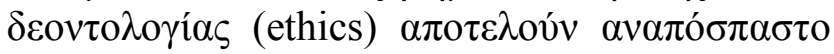

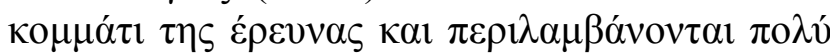

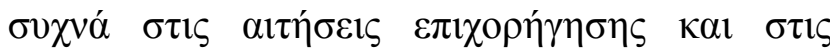

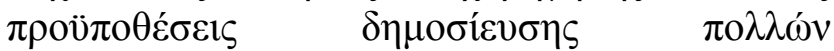

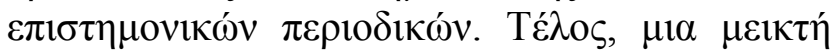

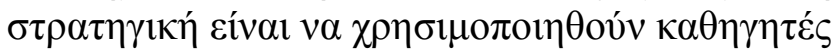

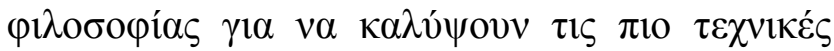

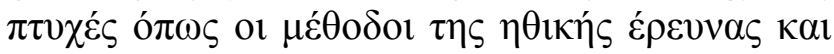

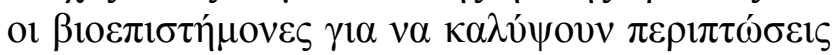

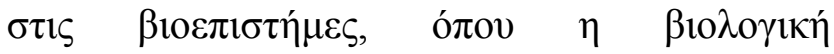

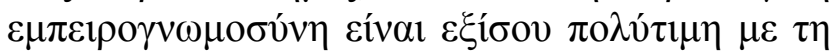

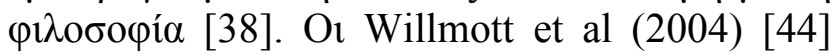

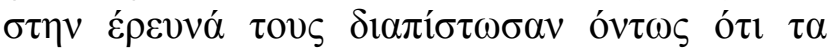
$\pi \varepsilon \rho 1 \sigma \sigma o ́ \tau \varepsilon \rho \alpha \quad \pi \rho о \gamma \rho \alpha ́ \mu \mu \alpha \tau \alpha \quad \sigma \pi о \nu \delta \omega ́ v$ 
$\beta 10 \varepsilon \pi \imath \sigma \tau \eta \mu \omega ́ v \quad \pi$ ov $\pi \varepsilon \rho \imath \lambda \alpha \mu \beta \alpha ́ v o v v \quad \sigma \eta ́ \mu \varepsilon \rho \alpha$

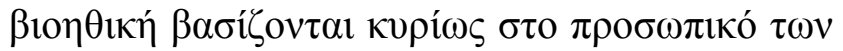

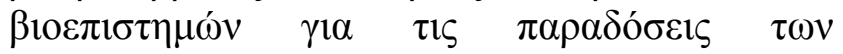

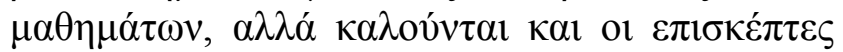

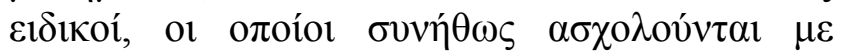

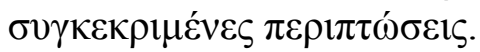

\section{$\Sigma v \mu \pi \varepsilon \rho \alpha ́ \sigma \mu \alpha \tau \alpha$}

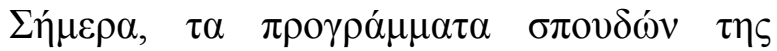

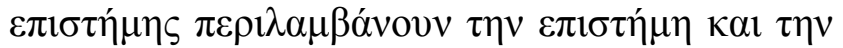

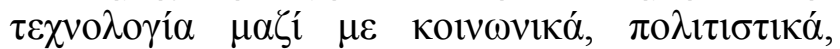

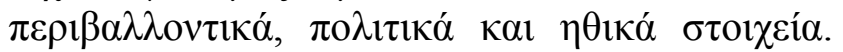

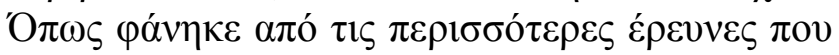

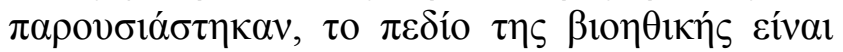

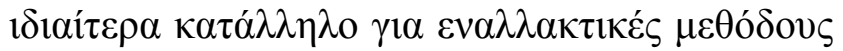

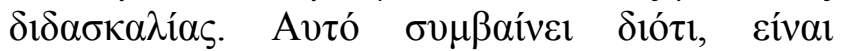

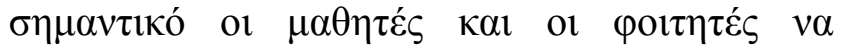

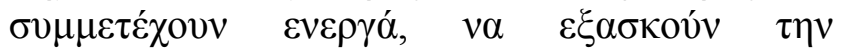

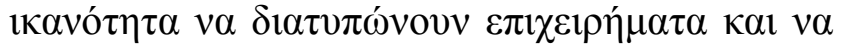

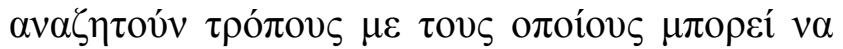

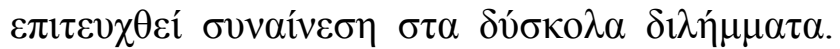

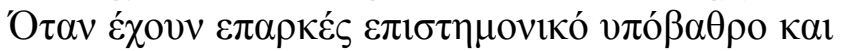

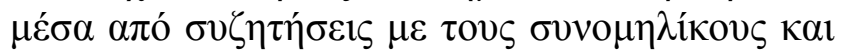

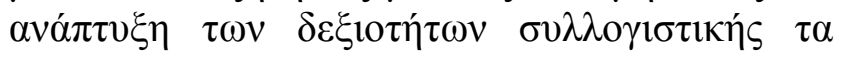

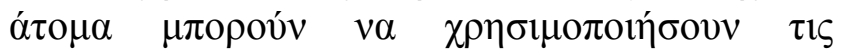

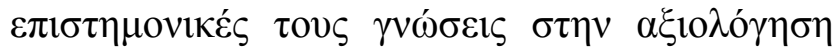

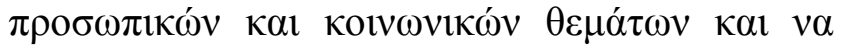

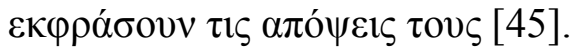

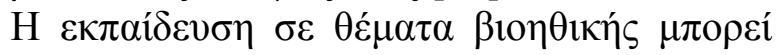

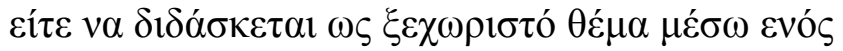

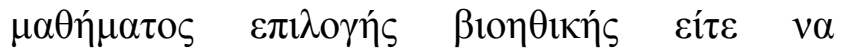
$\varepsilon v \sigma \omega \mu \alpha \tau \omega ́ v \varepsilon \tau \alpha 1 \quad \sigma \varepsilon \quad \alpha ́ \lambda \lambda \alpha \quad \mu \alpha \theta \eta \dot{\mu} \mu \alpha \alpha . \quad \Sigma \tau \eta$ $\delta \varepsilon v \tau \varepsilon \rho о \beta \alpha ́ \theta \mu 1 \alpha \quad \varepsilon \kappa \pi \alpha i ́ \delta \varepsilon v \sigma \eta \quad \pi \rho о \kappa \rho i ́ v \varepsilon \tau \alpha 1 \quad \eta$

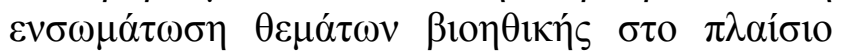

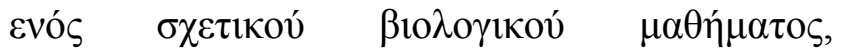

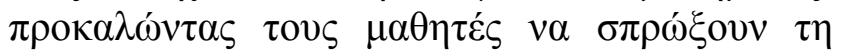

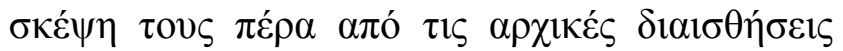

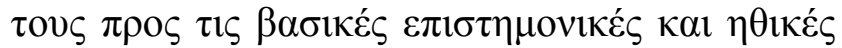

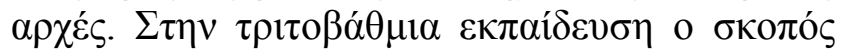

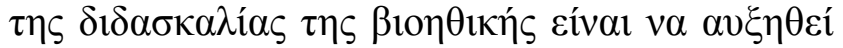

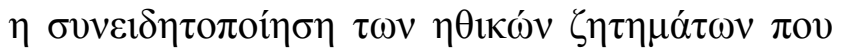

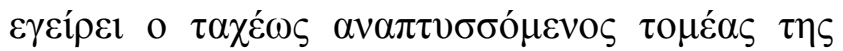

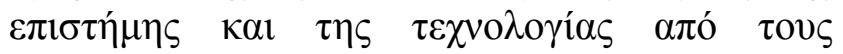

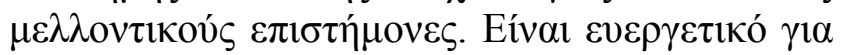

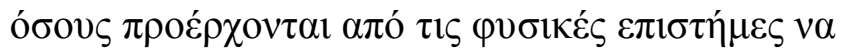

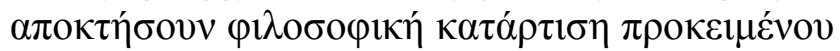

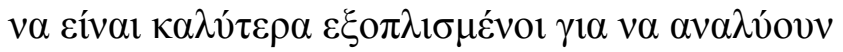

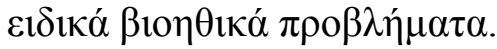

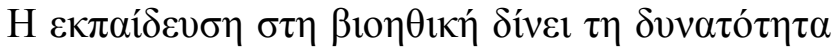

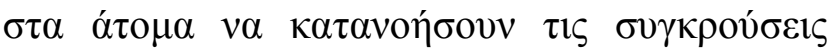

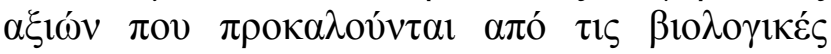

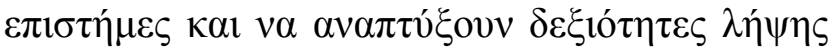

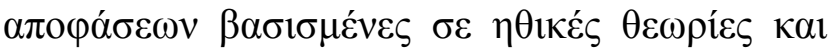

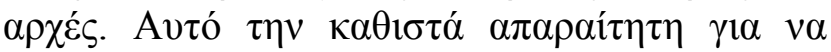

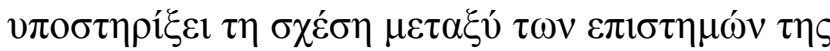

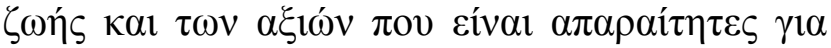

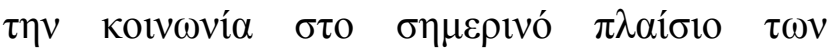

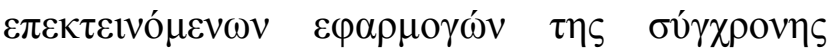

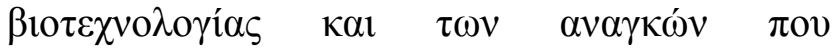

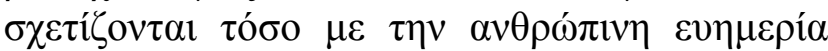

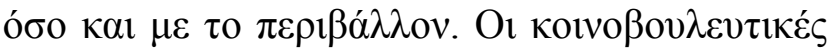

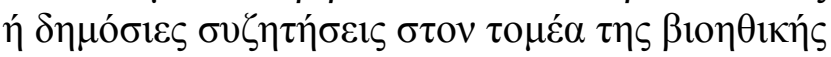

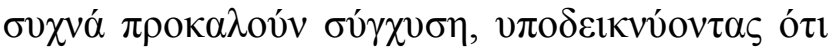

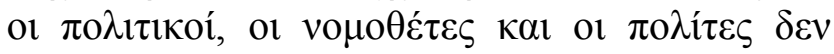

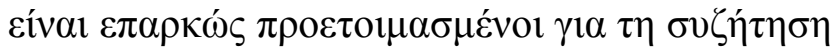

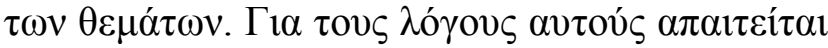

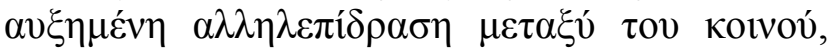

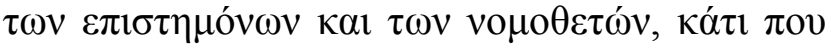

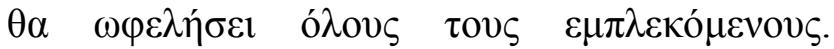

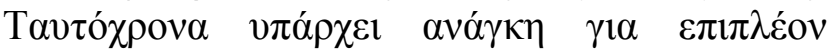

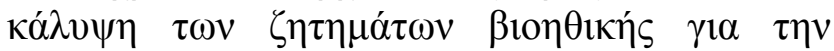

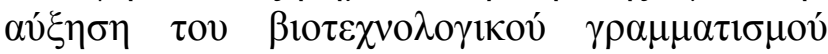

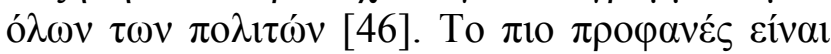

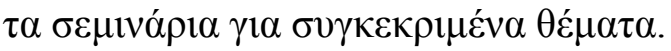

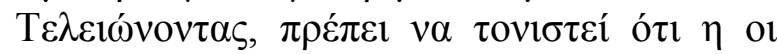

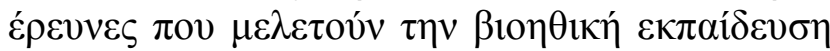

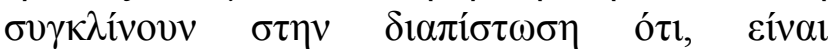

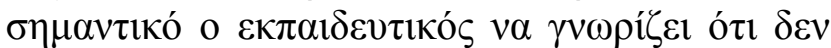

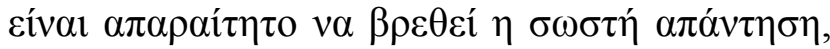

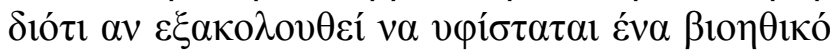

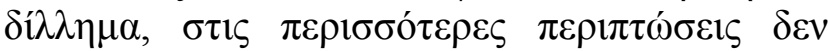

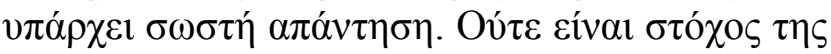

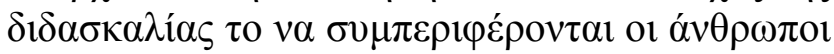

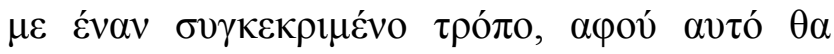

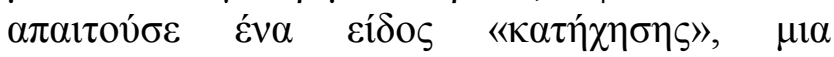

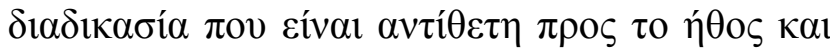

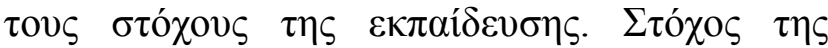
$\beta 10 \eta \theta 1 \kappa \eta ́ \varsigma \varepsilon \varepsilon \kappa \pi \alpha i ́ \delta \varepsilon v \sigma \eta \varsigma, \sigma o ́ \mu \varphi \omega v \alpha \mu \varepsilon \tau \eta v$ Iancu

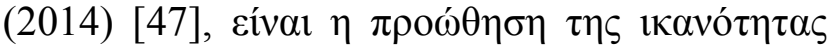

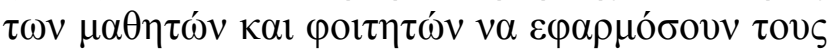

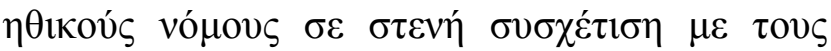

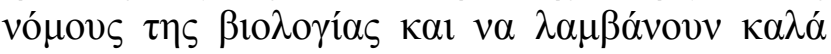

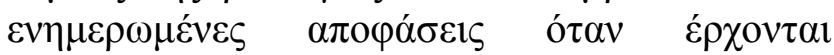

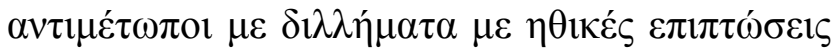

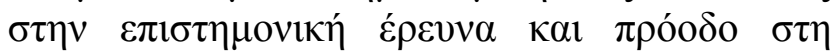

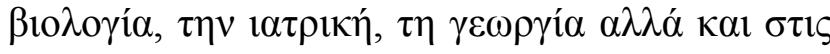

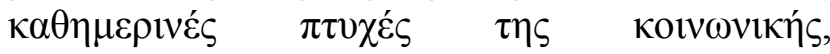

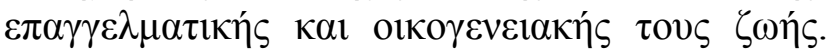




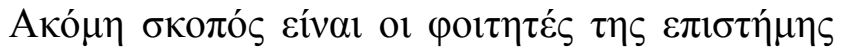

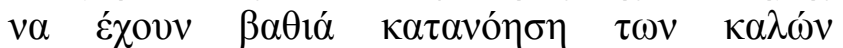

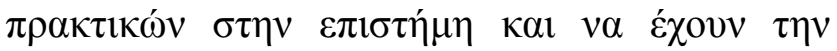

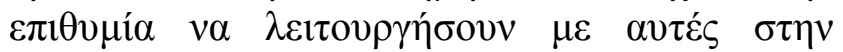

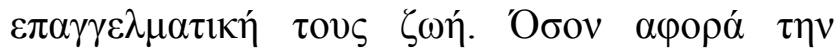

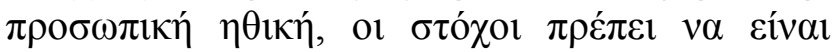

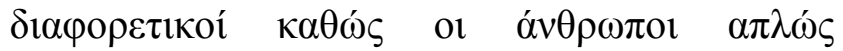

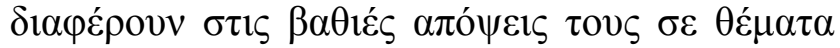

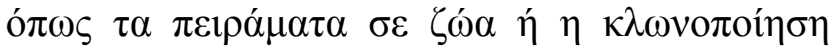

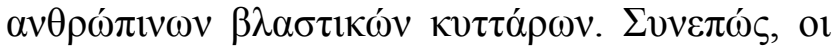

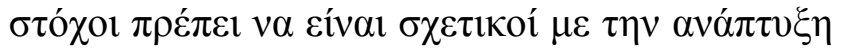

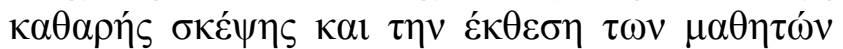

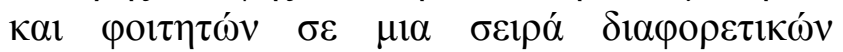
$\alpha \pi \delta ́ \psi \varepsilon \omega v$.

\section{Eлі́̉}

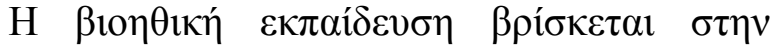

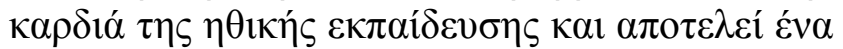

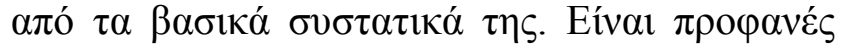

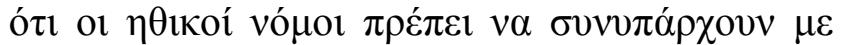

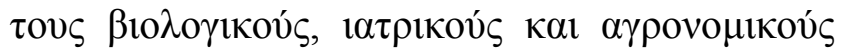
vó

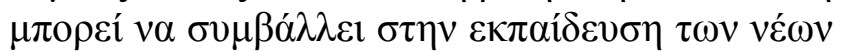

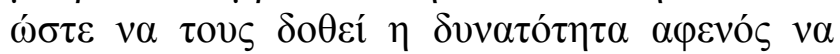

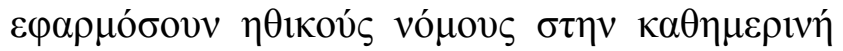

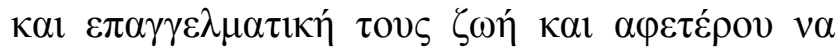

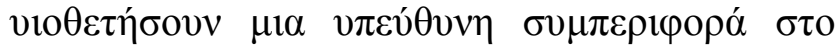

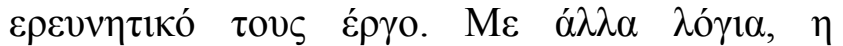

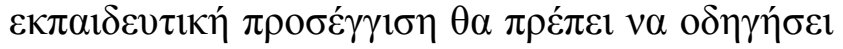

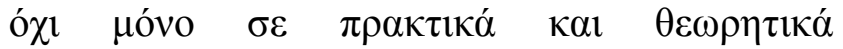

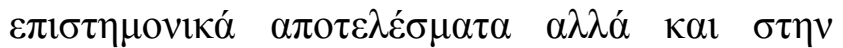

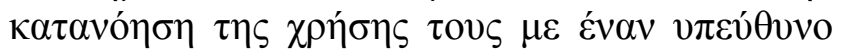

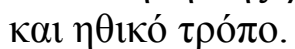

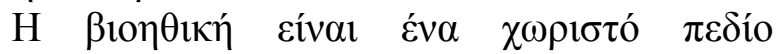

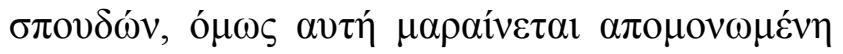

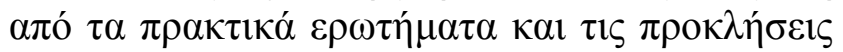

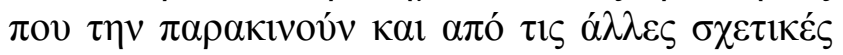

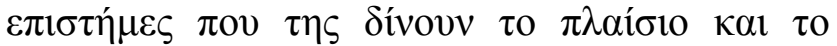

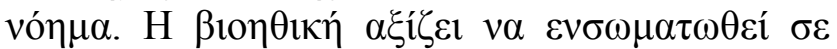

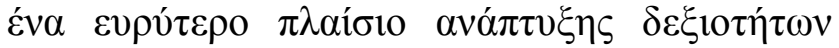

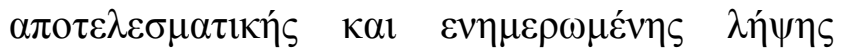

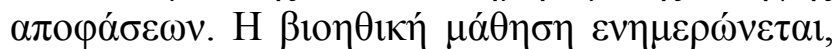

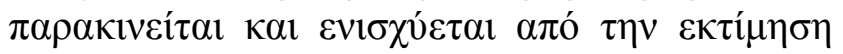

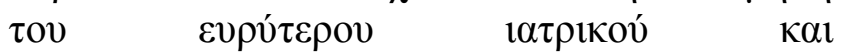

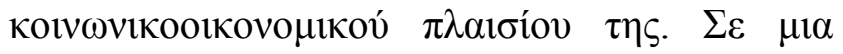

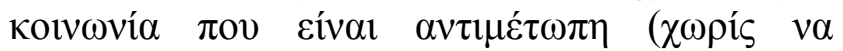
$\alpha \nu \tau \imath \mu \varepsilon \tau \omega \pi i \zeta \varepsilon \imath ;) \mu \varepsilon \tau \eta \nu \alpha v \alpha ́ \gamma \kappa \eta v \alpha \beta \varepsilon \lambda \tau i \omega ́ \sigma \varepsilon \imath \tau \eta \nu$

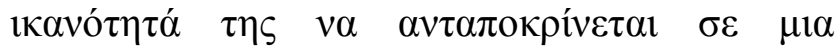

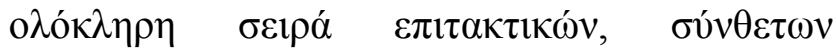

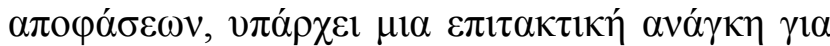

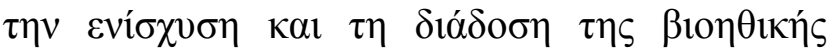
$\varepsilon \kappa \pi \alpha i ́ \delta \varepsilon v \sigma \eta \varsigma$.

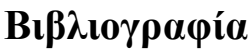

1. Steger F. Fritz Jahr's (1895-1953) European concept of bioethics and its application potential. European Journal of Bioethics, 2015, $6 / 2$.

2. Macer DRJ. Bioethics for the People by the People. Christchurch, N.Z.: Eubios Ethics Institute, 1994.

3. Have H. The Activities of UNESCO in the Area of Ethics. Kennedy Institute of Ethics Journal, The Johns Hopkins University Press, 2006, 16: 4: 333-351,.

4. Have H, Gordijn B. Broadening education in bioethics. Medicine Health Care and Philosophy, 2012, 15: 99.

5. Have H. Potter's notion of bioethics. Kennedy Inst Ethics J, 2012: 59-82.

6. Booth J, Garrett J. Instructors' Practices in and Attitudes Toward Teaching Ethics in the Genetics Classroom. Genetics, 2004: 11111117.

7. Downie R. The teaching of bioethics in the higher education of biologists. Journal of Biological Education, 1993: 34-48.

8. Sadler T, Zeidler $D$. The morality of socioscientific issues: Construal and resolution of genetic engineering dilemmas. Science Education, 2004: 4-27.

9. Bishop LJ, Szobota L. Teaching Bioethics at the Secondary School Level. Hastings Center Report, 2015: 19-25.

10. Macer D. Bioethics education for informed citizens across cultures. School Science Review, 2004: 83-86.

11. Macer D. Where do we learn bioethics and avenues for bioethics education. Proceedings of the UNESCO International Bioethics Committee Sixth Session, UNESCO, Paris, 2000, II: 25-32.

12. ROSE project, The Relevance of Science Education. English publications, 2009. Available at: https://roseproject.no/publications/englishpub.html. 
13. Reiss M. Ethical thinking. In: Reiss M, Jones A, McKim A (eds) Ethics in the Science and Technology Classroom: A New Approach to Teaching and Learning, Sense Publishers, Rotterdam, 2010: 7-17.

14. Osborne J, Collins S. Pupils' and Parents' Views of the School Science Curriculum. King's College London, London, 2000.

15. Kabir A, Imam M. Understanding lessons of ethics in the primary education of Bangladesh. Bangladesh Bioethics Society, 2010, 1: 3: 47-51.

16. Jones A, McKim A, Reiss M, Ryan B, Buntting C, Saunders K, De Luca R, Conner, L. Research and Development of Classroombased Resources for Bioethics Education in New Zealand, Hamilton: Wilf Malcolm Institute of Educational Research School of Education, University of Waikato, 2007.

17. Reiss M, Assessing ethics in secondary science. School Science Review, 2011, 93: 342.

18. Fischer ML, Cunha TR, Roth ME, Martins GZ. Path of Dialogue: a bioethics experience in primary school. Revista Bioética, 2017, 25: 1: 89-100.

19. Solomon MZ, Vannier D, Chowning JT, Miller JS, Paget KF. Pedagogical Challenges of Teaching High School Bioethics: Insights from the Exploring Bioethics Curriculum, Hastings Center Report, 2016, 46: 1: 11-18.

20. Gutierez S. Effects of Bioethics Integration on the Critical Thinking and DecisionMaking Skills of High School. International Journal of Learning, Teaching and Educational Research, 2014: 32-42.

21. Keskin-Samanci N, Özer-Keskin M, Arslan O. Development of 'Bioethical values inventory' for pupils in secondary education within the scope of bioethical education. Eurasia Journal of Mathematics, Science \& Technology Education, 2014: 69-76.

22. Nunes R, Duarte I, Santos C, Rego G. Education for values and bioethics. Springer Plus, 2015, 4: 45.

23. Fulda KG, Lykens K. Ethical issues in predictive genetic testing: a public health perspective. Journal of Medical Ethics, 2006: 143-147.

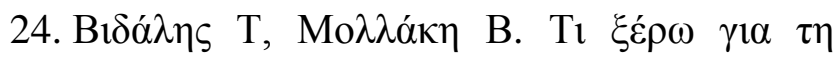

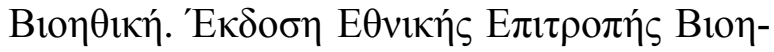

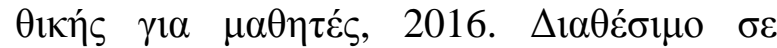
http://www.bioethics.gr/images/pdf/Booklet_ for_schools_final.pdf.

25. Ike CG, Anderson N. A proposal for teaching bioethics in high schools using appropriate visual education tools. Philosophy Ethics and Humanities in Medicine, 2018, 3:11.

26. Rivers SE, Reyna VF, Mills B. Risk taking under the influence: a fuzzy-trace theory of emotion in adolescence. Dev Rev, 2008, 28: 107-144.

27. Chowning JT, Griswold JC, Kovarik DN, Collins LJ. Fostering Critical Thinking, Reasoning and Argumentation Skills through Bioethics Education. PLoS ONE, 2012.

28. Asada Y, Tsuzuki M, Akiyama S, Macer NY, Macer D. High School Teaching of Bioethics in New Zealand, Australia and Japan. Journal of Moral Education, 1996: 401-420.

29. Matthiessen L. Education in bioethics: initiatives of the European Union. Teaching bioethics Report from a seminar, 2001: 95 - 106.

30. Ezer T, Overall J, Advancing human rights in patient care through education in Eastern Europe and Central Asia. Health Hum Rights, 2013, 15: 54-68.

31. Klapatyi D, Senyta I, Bozikov J, Teaching Human Rights in Patient Care: Law, Medicine and Public Health Faculties. The European Journal of Public Health, 2014: 24.

32. Chamberlain M, Human rights education for Nursing Students. Nursing Ethics, 2001: 211222.

33. Busquets E, Roman B, Terribas N. Bioethics in Mediterranean culture: the Spanish experience. Medicine Health Care and Philosophy, 2012, 15: 437-451.

34. Park S, Yoo SY. Effects of constructivist teaching methods on Bioethics education for nursing students: A quasi-experimental study. Nurse Education Today, 2014.

35. Casini M, Meaney J, Midolo E, Čartolovni A, Sacchini D, Spagnolo A. Why teach "Bioethics and Human Rights" to healthcare professions undergraduates? JAHR, 2014, 5: 2: 349-368. Available at https://hrcak.srce.hr/134786.

36. Have H, Gordijn B. Broadening education in bioethics. Medicine Health Care and Philosophy, 2012: 99-101. 
37. Booth JM, Garrett JM. Instructors' practices in and attitudes toward teaching ethics in the genetics classroom. Genetics, 2004, 168: 3: 1111-7.

38. Downie R, Clarkeburn H. Approaches to the teaching of bioethics and professional ethics in undergraduate courses. Bioscience Education, 2015: 1-9.

39. Bryant JA, Baggott la Velle LM. A bioethics course for biology and science education students. Journal of Biological Education, 2003, 37: $91-5$.

40. Jagger K, Furlong J. Infusing Bioethics into Biology and Microbiology Courses and Curricula: A Vertical Approach. Journal of Microbiology \& Biology Education, 2014: 213217.

41. Loike JD, Rush BS, Schweber A, Fischbach RL. Lessons learned from undergraduate students in designing a science-based course in bioethics. CBE Life Science Education, 2013: 701-10.

42. Illingworth S. Approaches to ethics in higher education. Philosophical and Religious Studies Subject Centre, LTSN, University of Leeds, 2004. Available at http://aulre.org/heaprsarchive/sec2_resources _ethics/5_across_curriculum.pdf
43. Smith S, Fryer-Edwards K, Diekema DS, Braddock $\mathrm{CH}$ 3rd. Finding effective strategies for teaching ethics: a comparison trial of two interventions. Academic Medicine, 2004: 265-71.

44. Willmott CJR, Bond AN, Bryant JA, Maw SJ, Sears HJ, Wilson JM. Teaching ethics to bioscience students - a survey of undergraduate provision. Bioscience Education EJournal, 2004: 3-9.

45. Sadler TD, Donnelly LA. Socioscientific argumentation: the effects of content knowledge and morality. International Journal of Science Education, 2006: 1463-1488.

46. Klungland $\mathrm{H}$. Teaching bioethics group report and final discussions. Teaching bioethics Report from a seminar, 2001: 135- 141.

47. Iancu M. Bioethical education in teaching Biology. Procedia - Social and Behavioral Sciences, 2014: 73-77. 